Mathématiques et sciences humaines
Mathematics and social sciences

148 | Hiver 1999

Varia

\title{
Buffon et l'arithmétique politique
}

Buffon and the political arithmetic

\section{Thierry Martin}

\section{(2) OpenEdition}

Journals

Édition électronique

URL : http://journals.openedition.org/msh/2805

DOI : $10.4000 /$ msh.2805

ISSN : 1950-6821

\section{Éditeur}

Centre d'analyse et de mathématique sociales de l'EHESS

\section{Édition imprimée}

Date de publication : 1 décembre 1999

ISSN : 0987-6936

\section{Référence électronique}

Thierry Martin, «Buffon et l'arithmétique politique », Mathématiques et sciences humaines [En ligne], 148 | Hiver 1999, mis en ligne le 10 février 2006, consulté le 23 juillet 2020. URL : http:// journals.openedition.org/msh/2805; DOl : https://doi.org/10.4000/msh.2805 
Math. Inf. Sci. hum., (37 année, ${ }^{\circ} 148,1999$, pp. 5-30)

\title{
BUFFON ET L'ARITHMÉTIQUE POLITIQUE
}

\author{
Thierry MARTIN ${ }^{1}$
}

\begin{abstract}
RÉSUMÉ - L'objet de cette analyse est d'étudier les contributions de Buffon à l'arithmétique politique, envisagées non pas du point de vue de la constitution historique et technique de la démographie, mais en les replaçant dans le projet qui commande la réflexion de Buffon. S'efforcer de restituer ainsi à ces travaux leur véritable sens permet non seulement de comprendre la disparité des jugements qu'ils ont suscités, mais aussi d'éclairer quelques-unes des difficultés qu'ils recèlent, et de faire apparaître une évolution qui semble se dessiner dans la relation de Buffon à la mathématisation du social.

MOTS-CLÉS - Buffon, arithmétique politique, probabilités, démographie, mortalité.

SUMMARY - Buffon and the political arithmetic.

This analysis is aimed to study Buffon's contributions towards political arithmetic, considered not from the point of view of the historical and technical constitution of demography but by replacing them in the general proposal which commands Buffon's thought. The task for assign to these works their real meaning allows not only to understand the disparity of the judgements they raised up but to enlighten too some of the difficulties they conceal and to show an evolution, which seems to appear in Buffon's relationship to the mathematisation of social sciences.
\end{abstract}

KEYWORDS - Buffon, political arithmetic, probability, demography, mortality.

Cette analyse prend appui sur un double constat : d'un côté le nom de Buffon est fréquemment invoqué dans les études consacrées à l'arithmétique politique, mais de l'autre ses travaux suscitent des jugements contrastés sur leur intérêt, élogieux par exemple chez Fréchet ${ }^{2}$ ou Lucien Badet ${ }^{3}$, sévères, au contraire, de la part de J. Dupâquier ${ }^{4}$ ou L. Behar ${ }^{5}$. Ce constat invite à repérer dans l'œuvre de Buffon les textes qui peuvent

\footnotetext{
1 Université de Franche-Comté, 30 rue Mégevand, 25030 BESANÇON Cedex, e-mail: thierry.martin@univ-fcomte.fr

${ }^{2}$ Maurice Fréchet, «Buffon, philosophe des mathématiques», Bulletin de l'institut d'Égypte, n 28,1954 , pp. 185-202, puis «Buffon comme philosophe des mathématiques», CEuvres philosophiques de Buffon, édité par Jean Piveteau, Paris, P.U.F., 1954, pp.435-446; repris sous le titre «Buffon comme mathématicien, statisticien et philosophe des mathématiques» dans Les mathématiques et le concret, Paris, P.U.F., 1955, pp. 344-367.

${ }^{3}$ Lucien Badet, «Buffon, précurseur de la science démographique», Annales de géographie, tome XXXVII, 1929, pp. 206-220.

${ }^{4}$ J. et M. Dupâquier, Histoire de la démographie. La statistique de la population des origines à 1914, Paris, Librairie académique Perrin, 1985, chapitres 5 et 6.

${ }^{5}$ Lazare Behar, «Des tables de mortalité aux XVII et XVIII ${ }^{e}$ siècles. Histoire - Signification», Annales de démographie historique, 1976, pp. 173-200.
} 
constituer des contributions à l'arithmétique politique, en donnant de celle-ci une définition suffisamment large, telle que celle proposée par l'Encyclopédie méthodique sous la plume de Diderot, pour qui elle désigne cette branche des mathématiques qui a «pour but des recherches utiles à l'art de gouverner les peuples» ${ }^{6}$, ou sous celle de Condorcet pour qui elle consiste en «l'application du calcul aux sciences politiques» ${ }^{7}$, pour les soumettre à l'examen. Celui-ci se propose d'interroger non pas l'intérêt technique ou historique de ces textes - les auteurs précédemment cités s'en sont déjà chargés -, mais le sens que leur assigne le projet qui anime la réflexion de Buffon ; l'hypothèse initiale de cette étude étant justement que c'est en référence à ce projet de Buffon que peuvent se comprendre ces divergences d'appréciation.

Les travaux de Buffon susceptibles de relever de l'arithmétique politique sont essentiellement concentrés dans deux groupes de textes ${ }^{8}$ de volume très inégal, d'une part le chapitre intitulé «De la vieillesse et de la mort» appartenant à l'Histoire naturelle de l'homme, publiés en 1749 dans le tome II de l'Histoire naturelle, générale et particulière, de l'autre, la série des articles constituant le cour du Supplément à cette histoire naturelle de l'homme, soit le tome IV du Supplément à l'Histoire naturelle, publiés en 1777. Cette série d'articles est composée de l'Essai d'arithmétique morale, des Probabilités de la durée de la vie, puis d'un ensemble d'études des tables des naissances, mariages et morts de la ville de Paris et de plusieurs villes et villages de Bourgogne, enfin des comparaisons de la mortalité à Paris et dans les campagnes, en France et en Angleterre.

La situation de ces textes dans l'œuvre de Buffon appelle d'emblée deux remarques :

$1^{\circ}$ ) Ils relèvent à ses yeux de l'histoire naturelle, ou du moins peuvent légitimement y être intégrés. Ce constat engendre une difficulté, car si ces travaux s'inscrivent effectivement dans le projet d'une histoire naturelle, leur appartenance à l'arithmétique politique devient problématique. Une première question est ainsi soulevée : en quel sens ces textes appartiennent-ils à l'histoire naturelle, et corrélativement peuvent-ils être considérés comme des contributions à l'arithmétique politique?

$2^{\circ}$ ) Ces textes sont reliés les uns aux autres, mais de manière fort lâche. D'une part, les Probabilités de la durée de la vie, bien que publiées près de trente ans après l'article De la vieillesse et de la mort, sont construites à partir de la table de mortalité de Dupré de SaintMaur que donnait Buffon à la fin de cet article, et donc semblent venir prolonger et achever l'analyse de 1749. Mais immédiatement, deux différences s'imposent au premier regard :

- Les Probabilités de la durée de la vie s'étendent sur plus de cent pages - elles couvrent à elles seules les pages 149 à 264 du tome IV du Supplément, auxquelles s'ajoutent la soixantaine de pages consacrées aux tables de naissances, mariages et

\footnotetext{
${ }^{6}$ Encyclopédie méthodique, Mathématiques, Paris, Panckoucke, t. I, 1784, p. $132 \mathrm{~b}$.

${ }^{7}$ Ibid., p. 135 a.

${ }^{8}$ Les deux mémoires, Sur la conservation et le rétablissement des forêts de 1741 et Sur la culture et l'exploitation des forêts de 1742, qui auraient pu relever de l'arithmétique politique, dans la mesure où ils contiennent des développements relatifs à ce qu'on appellerait aujourd'hui l'aménagement du territoire et où la culture, l'entretien et le développement des forêts est un sujet qui intéresse directement l'administration du royaume (puisque, le bois servant à la construction des navires, la culture des forêts est une condition du développement maritime), s'en tiennent à une analyse descriptive, dépourvue de tout traitement mathématique.
} 
morts $^{9}$ - tandis que l'analyse de la table de mortalité de Dupré de Saint-Maur n'occupait en 1749 qu'une quinzaine de pages ${ }^{10}$,

- Le calcul des probabilités de durée de vie se réduisait en 1749 à un unique tableau, succédant à la présentation détaillée de la table de Dupré de Saint-Maur, tandis qu'inversement, en 1777, cette table, condensée car ne détaillant pas les décès par communes, est suivie de la longue série des calculs probabilistes.

D'autre part, l'Essai d'arithmétique morale, les Probabilités de la durée de la vie et les études démographiques qui les suivent forment un ensemble d'analyses partageant leur commun recours à l'instrument mathématique, enchâssées dans le tome IV du Supplément entre une série de réponses à des objections et une série d'additions aux articles des tomes II et III de l'Histoire naturelle qui ont un tout autre objet. Mais, en même temps, ces différents textes sont simplement juxtaposés, sans relation organique explicite.

Une double question est ainsi soulevée à propos de l'articulation de ces textes les uns par rapport aux autres :

- Celle de la relation qu'entretient l'Essai d'arithmétique morale avec les travaux de Buffon sur la mortalité, autrement dit celle de la relation de l'arithmétique morale à l'arithmétique politique,

- Celle de l'évolution de la pensée de Buffon : y a-t-il continuité ou rupture dans la méthode mise en œuvre par Buffon, comme dans les préoccupations qui l'animent, entre 1749 et 1777 ?

\section{DE LA VIEILLESSE ET DE LA MORT}

L'analyse de Buffon dans l'article De la vieillesse et de la mort, qui clôt le tome II de l'Histoire naturelle ${ }^{11}$, se déploie à deux niveaux distincts, l'essentiel du texte étudiant le dépérissement puis la mort de l'homme d'un point de vue physiologique, les dernières pages étant, quant à elles, consacrées à l'analyse des probabilités de durée de vie, telles qu'on peut les calculer à partir de la table de mortalité construite par Dupré de Saint-Maur.

Si on laisse provisoirement de côté ces dernières pages, l'article développe une analyse du vieillissement humain fondée sur une analogie avec le développement et le dépérissement des arbres, dont on sait qu'ils furent l'objet d'un ensemble de mémoires présentés par Buffon à l'Académie des Sciences dans les années 1737-1742 ${ }^{12}$, analogie faisant apparaître le vieillissement comme une solidification et un assèchement progressifs des composants du corps vivant. L'analyse conduit à établir, relativement à la mortalité, deux propositions générales.

\footnotetext{
${ }^{9}$ Histoire naturelle, générale et particulière, servant de suite à l'Histoire naturelle de l'Homme, Supplément, tome quatrième, Paris, Imprimerie Royale, 1777, pp. 265 à 323 du volume.

${ }^{10}$ Pages 588 à 603 du tome II de l'Histoire naturelle, générale et particulière, Paris, Imprimerie royale, 1749.

${ }^{11}$ Histoire naturelle, générale et particulière, Paris, Imprimerie royale, tome second, 1749, pp. 557-603.

${ }^{12}$ Mémoires repris dans le tome II du Supplément à l'Histoire naturelle (1775). (Ils sont l'objet de l'étude menée par Lesley Hanks aux pages 141-213 de Buffon avant l' «Histoire Naturelle », Paris, P.U.F., 1966).
} 
- La première pose l'existence d'une relation de proportionnalité entre temps de croissance et longévité, relation qui vaut pour l'ensemble du monde vivant, aussi bien végétal qu'animal. «La durée de la vie dit Buffon est proportionnelle au temps de l'accroissement» ${ }^{13}$, étant entendu que par accroissement, il faut comprendre le développement complet de l'individu, et non pas seulement sa croissance en taille. Appliquée à l'homme, cette loi signifie que son développement complet n'est achevé qu'à trente ans, même si sa taille adulte est atteinte vers 16 ou 18 ans, et sa «durée de vie» est de 90 à 100 ans $^{14}$.

- Cette première loi en engendre une seconde affirmant l'uniformité de la durée de vie. En effet, posée comme une donnée physiologique, la relation de proportionnalité précédente vaut donc pour tous les individus de la même espèce. Il en résulte l'unité de l'espèce humaine et par conséquent l'uniformité de la durée de vie des individus qui la composent. Buffon écrit ainsi : «à prendre le genre humain en général, il n'y a, pour ainsi dire, aucune différence dans la durée de la vie» ${ }^{15}$, ce qu'il explicite en affirmant son indépendance à l'égard des conditions sociales d'existence des individus : «la durée de la vie ne dépend ni des habitudes, ni des mœurs, ni de la qualité des aliments». Et il insiste avec force sur cette uniformité, précisant que «l'Européen, le Nègre, le Chinois, l'Américain, l'homme policé, l'homme sauvage, le riche, le pauvre, l'habitant de la ville, celui de la campagne, si différents entre eux par tout le reste, se ressemblent à cet égard, et n'ont chacun que la même mesure, le même intervalle de temps à parcourir depuis la naissance à la mort ; [...] la différence des races, des climats, des nourritures, des commodités n'en fait aucune à la durée de la vie» ${ }^{16}$. Les seules différences notables dans la durée de vie ne pourront donc provenir que de caractères physiques ou physiologiques, à l'exception des facteurs sociaux. C'est effectivement ce qu'indique Buffon, relevant comme seules différences de durée de vie :

$\left.1^{\circ}\right)$ La différence de longévité entre l'homme et la femme, dont Buffon précise qu'elle est attestée par les tables de mortalité, et qui s'explique, puisque la vieillesse est un processus de solidification, par la plus grande souplesse des différentes parties du corps féminin par rapport au corps masculin,

$2^{\circ}$ ) Les variations dues à la qualité de l'air : la vieillesse étant une perte de souplesse et d'élasticité, un air plus dense, plus lourd, comme celui des vallées peut, selon Buffon, abréger la durée de vie, tandis que l'air des montagnes favorisera, au contraire, la longévité.

La fin de l'article, consacrée à l'étude de la table de mortalité de Dupré de SaintMaur, s'ouvre sur une formule dont la signification est d'abord obscure, «après avoir fait l'histoire de la vie et de la mort par rapport à l'individu, considérons l'une et l'autre dans l'espèce entière ${ }^{17}$, et, apparemment, ne s'accorde pas avec l'analyse précédente. En effet, la table de Dupré de Saint-Maur permet d'établir, écrit Buffon, «qu'à l'âge de 28 ou 29 ans on a vécu la moitié de sa vie» ${ }^{18}$, elle semble donc contredire la première loi. De plus, Buffon justifie son choix de la table de Dupré de Saint-Maur comme table de référence (laquelle, d'ailleurs, ne distingue pas les décès selon le sexe) en arguant du fait que les autres tables ne concernent que des catégories particulières d'individus, à savoir les

\footnotetext{
${ }^{13}$ «De la vieillesse et de la mort», Histoire naturelle, tome II, 1749, p. 573.

${ }^{14}$ Ibid., p. 569.

${ }^{15}$ Ibid., p. 572.

${ }^{16}$ Ibid., p. 571.

${ }^{17}$ Ibid., p. 588.

${ }^{18}$ Ibid., p. 602.
} 
citadins pour les tables de Halley, Graunt, Kerseboom ou Sympson, les rentiers et les ecclésiastiques pour Deparcieux, montrant par là qu'il admet implicitement que la durée de vie varie avec les conditions sociales des individus.

Cet apparent désaccord s'explique lorsqu'on comprend que par «durée de vie», le début de l'article entend la longévité maximale dont est susceptible l'individu, tandis que la même expression désigne ensuite la durée moyenne de vie telle qu'elle est révélée par les données statistiques. Il ne s'agit donc pas seulement, comme l'annonçait la phrase d'ouverture, de passer de la considération de l'individu à celle de l'espèce, mais il y a, entre le corps de l'article et sa fin, à la fois changement d'objet et changement de plan : à l'étude de la durée maximale de la vie individuelle succède l'analyse de la mortalité moyenne observée aux différents âges, et conjointement aux considérations physiologiques est substituée une analyse statistique.

Et il semble bien qu'alors la préoccupation de Buffon relève de l'arithmétique politique, puisque la nécessité d'établir «quelque chose de fixe sur la mortalité des hommes à différents âges» prend appui sur l'affirmation selon laquelle «si ces observations étaient assez exactes et assez multipliées, elles seraient d'une très grande utilité pour la connaissance de la quantité du peuple, de sa multiplication, de la consommation des denrées, de la répartition des impôts, etc.» ${ }^{19}$.

Cependant, opposer ainsi l'analyse à caractère physiologique de la longévité maximale, insistant sur l'unité de l'espèce humaine et son uniformité, et la perspective socio-politique des dernières pages de l'article, c'est manquer le sens du discours de Buffon. S'il fonde, en effet, son analyse sur la table de mortalité de Dupré de Saint-Maur, c'est, dit-il, parce qu'elle mobilise non seulement les registres des paroisses de Paris, «où il entre des étrangers et d'où il sort des natifs, mais encore ceux des campagnes, afin qu'ajoutant ensemble tous les résultats, les uns compensent les autres ${ }^{20}$, autrement dit parce qu'elle est la seule qui permette de mesurer «des probabilités exactes sur la durée générale de la vie», id est sur celle «des hommes en général». Faire porter l'analyse sur l'homme en général, c'est lui assigner comme objet la construction de probabilités de durée de vie valant pour l'espèce, dépassant par conséquent les différences relatives au sexe, aux catégories sociales, aux situations géographiques, alors que l'arithmétique politique s'emploiera, au contraire, à distinguer les variations de la mortalité en fonction de ces paramètres.

Il apparaît donc qu'en 1749, si l'analyse menée par Buffon sur la mortalité peut fournir des éléments à l'arithmétique politique, son projet est avant tout anthropologique. Il s'agit d'une arithmétique naturaliste, et non d'une arithmétique politique. Et c'est bien ce qu'indiquait la phrase d'ouverture se proposant d'étudier la mortalité au niveau de «l'espèce entière». Il n'est pas étonnant dans ces conditions que Buffon ne prenne pas la peine de préciser dans la table de mortalité les années de référence ayant permis de comptabiliser les décès, pas plus qu'il ne tienne compte des naissances intervenues au cours de ces années (cf. annexe 1 en fin d'article), son but n'étant pas ici de faire œuvre de démographe.

En conséquence, s'il y a bien, entre le début et la fin du texte, un changement d'objet, et si l'argumentation ne mobilise plus les mêmes instruments, son orientation, en revanche, demeure la même : il s'agit de penser la mortalité au niveau de l'espèce, donc de l'homme en général, indépendamment de ses conditions particulières d'existence. Nous

\footnotetext{
${ }^{19}$ Ibid., p. 588.

${ }^{20}$ Ibid., p. 589.
} 
retrouvons ainsi les analyses de Lazare Behar, qui voit dans ce double caractère (prétention à l'universalité et soumission à des préoccupations extra démographiques) l'un des traits dominants des tables de mortalité au XVIII ${ }^{\mathrm{e}}$ siècle, particulièrement net également, mais dans un sens tout différent, chez Süßmilch, et attestant, selon lui, de «la présence, à l'arrière-plan, de l'idéologie humaniste et universaliste «propre au Siècle des Lumières» ${ }^{21}$. Mais on peut préciser que justement le poids de cette dimension universaliste tient chez Buffon essentiellement à ce que son projet n'est pas directement démographique, mais anthropologique, et ceci en un double sens, son analyse étant à la fois intégrée à une étude relevant de l'histoire naturelle et motivée par des considérations morales.

C'est ce que permet de vérifier le traitement auquel Buffon soumet la table de mortalité de Dupré de Saint-Maur. Celle-ci, construite grâce aux registres de trois paroisses parisiennes et de douze paroisses rurales, soit un effectif global de 23994 décès, lui permet de mesurer le temps «qu'on peut espérer raisonnablement» ${ }^{22}$ de vivre aux différents âges, calcul dont les résultats sont regroupés dans la «table des probabilités de la durée de la vie». Cette table (reproduite à l'annexe 2) et le commentaire qu'en propose Buffon appellent plusieurs remarques :

$1^{\circ}$ ) Buffon ne précise pas en détail le mode de calcul utilisé pour obtenir ces résultats. La seule indication à ce sujet tient dans l'observation suivante : «Par la table des paroisses de la campagne, il paraît que la moitié de tous les enfants qui naissent meurent à peu près avant l'âge de quatre ans révolus ; par celle des paroisses de Paris, il paraît au contraire qu'il faut seize ans pour éteindre la moitié des enfants qui naissent en même temps» ${ }^{23}$. Est ainsi mentionné le principe directeur du calcul, à savoir un calcul de «vies médianes» ou «vies probables» ${ }^{24}$, expressions que n'utilise pas Buffon, parlant de «probabilités de la durée de la vie» ou encore de «l'âge auquel on peut espérer une plus longue durée de vie». Mais, si le mode de calcul des années de vie probable est ainsi donné, celui des mois reste indéterminé. Une vérification des calculs de vies probables (cf. annexe 3) fait apparaître des résultats (colonnes $\mathrm{E}$ et $\mathrm{F}$ ) très voisins de ceux proposés par Buffon (colonnes $\mathrm{G}$ et $\mathrm{H})$, les écarts ne portant que sur les mois et s'avérant généralement faibles. Leur irrégularité, cependant, dissimule la méthode utilisée par Buffon, dont on peut alors dire qu'elle est sans doute triviale, mais recourt à une technique d'approximation qui demeure obscure.

$2^{\circ}$ ) Ces «probabilités de durée de la vie» sont calculées pour l'ensemble des 15 paroisses, Buffon gommant du même coup la différence qu'il aperçoit entre la mortalité à Paris et celle de la campagne, (différence signalée dans le passage précédemment cité et due notamment à ce que de nombreux enfants parisiens sont mis en nourrice hors de la capitale).

$3^{\circ}$ ) Il assigne immédiatement à ses résultats une validité universelle, étendant à l'homme en général ce qu'il vient d'établir sur cet échantillon particulier : «on voit, par cette table, écritil, qu'on peut espérer raisonnablement, c'est-à-dire parier un contre un, qu'un enfant qui vient de naître ou qui a zéro d'âge, vivra huit ans ; qu'un enfant qui a déjà vécu un an ou qui a un an d'âge vivra encore trente-trois ans [...] qu'un homme de vingt ans révolus vivra encore trente-trois ans cinq mois... ${ }^{25}$.

\footnotetext{
${ }^{21}$ L. Behar, op. cit. supra note 4, p. 194.

${ }^{22}$ De la vieillesse et de la mort, in Histoire naturelle, tome II, 1749, p. 602.

${ }^{23}$ Ibid., p. 600.

${ }^{24}$ Il s'agit donc de déterminer, pour un âge donné, l'âge auquel l'individu a une chance égale de vivre ou de mourir, identifié au temps nécessaire à la disparition de la moitié de l'effectif des survivants à l'âge considéré.

${ }^{25}$ Ibid., p. 602 ; c'est nous qui soulignons.
} 
$4^{\circ}$ ) Enfin, tout en reconnaissant que l'on peut tirer de la table de Dupré de Saint-Maur «plusieurs connaissances utiles», il se borne à considérer «ce qui regarde les degrés de probabilité de la durée de la vie», et, de ces «degrés de probabilité», il ne retient que deux conséquences, savoir :

- Que l'âge où cette probabilité est la plus élevée est l'âge de 7 ans,

- Qu'il convient de tempérer les résultats fournis par le calcul par des «considérations morales», qui leur donnent leur sens «le plus réel». D'un point de vue physique, le calcul conduit à des vérités «mortifiantes», puisqu'il établit qu'à 12 ou 13 ans on a vécu le quart de sa vie, car on ne peut plus espérer que 38 ou 39 ans de plus; à 28 ou 29 , on en a vécu la moitié car notre «espérance» est réduite à 28 ans ; à 50 ans enfin, on a vécu les trois quarts de notre vie puisque notre espérance n'est plus que de 16 ou 17 ans. En quoi ces résultats peuvent-ils être compensés par des «considérations morales»? Buffon s'en explique en indiquant que «nous ne commençons à vivre moralement que quand nous commençons à ordonner nos pensées, à les tourner vers un certain avenir, et à prendre une espèce de consistance, un état relatif à ce que nous devons être dans la suite» ${ }^{26}$. La vie morale, c'est-à-dire pour l'homme la vie réelle, ne débute donc qu'à partir du moment où il est apte à se déterminer dans ses actions en anticipant l'avenir probable, par conséquent à partir du moment où il est à même de décider en considérant ses risques et ses espérances. L'âge moral est, dirait aujourd'hui Buffon, celui à partir duquel on est capable d'effectuer un choix rationnel. Buffon estimant cet âge à 15 ans, cela revient à considérer nos 15 premières années comme moralement nulles. Il en résulte, indique-t-il, qu'à 25 ans, on a vécu moralement le quart de notre vie, à 38 ans, la moitié, et à 56 seulement les trois quarts $^{27}$.

Finalement, à la question de savoir si Buffon fait ici œuvre d'arithméticien politique, on peut répondre par la négative. Il n'en a ni la démarche, ni le projet, puisque son but est essentiellement de dessiner la forme générale de la mortalité de l'espèce humaine et de mettre en évidence la nécessité de compenser cette donnée physique par le «point de vue moral».

C'est justement cette perspective morale qui commande le développement de l'Essai d'arithmétique morale, dont on peut se demander s'il vient infléchir l'analyse menée en 1749 en direction de l'arithmétique politique.

\section{L'ESSAI D'ARITHMÉTIQUE MORALE}

La structure du texte présente une grande complexité, dans la mesure où il s'agit d'un texte composite, où se mêlent des développements sur des sujets différents, composés à des dates parfois très éloignées, l'Essai étant notamment l'occasion pour Buffon de

\footnotetext{
${ }^{26}$ Ibid., pp. 602-603.

${ }^{27}$ Il suffit pour l'établir d'un calcul élémentaire que Buffon n'éprouve pas le besoin d'indiquer. En désignant par $p$ l'âge physique, et par $m$ l'âge moral, on a $m=p-15$. Pour un âge physique $p$, où l'on peut espérer vivre $n$ années, la durée de vie morale $d$ est égale à $m+n$, soit $(p-15)+n$. Pour $p=25, m$ $=10$, et puisque, d'après la table des probabilités de durée de vie, on peut espérer vivre encore 30 ans et 9 mois, arrondis à 31 ans, $d=31+10$, soit 41 ans. Tout se passe donc comme si l'on avait vécu 10 ans sur une vie totale de 41 ans. Pour $p=38, m=23$, et puisqu'on peut espérer environ 23 ans, $d=23+23$, soit 46 ans, et tout se passe comme si l'on avait vécu 23 ans sur une vie totale de 46 ans. Enfin, pour $p=56, m=41$, et puisqu'on peut encore espérer 13 ans, $d=41+13$, soit 54 . Tout se passe donc comme si l'on avait vécu 41 ans sur 54 .
} 
rassembler plusieurs mémoires antérieurement publiés ${ }^{28}$. Les passages les plus récents datent, semble-t-il, des années 1760-1764 ${ }^{29}$. Mais, on peut, par-delà l'organisation de détail, l'ordonner en deux grands moments, dont le premier étudie la mesure dans l'incertain et le second est consacré à une réflexion sur les mesures, mesures arithmétiques, géométriques puis physiques. Le premier moment se propose de montrer comment l'homme raisonnable doit estimer ses risques et ses espérances, et le second s'emploie à définir les conditions et limites de l'opération de mesure.

Il s'agit alors pour Buffon de penser ces deux objets du point de vue moral, c'est-àdire relativement aux décisions que la pratique nous impose de prendre, ou plus généralement relativement à leurs applications à la vie sociale. Il s'agit, plus précisément, de les soumettre à la juridiction de la raison pratique, dans la mesure où ils subissent le poids d'un double arbitraire, que Buffon entreprend de dénoncer et de réduire.

Le premier arbitraire tient à la représentation conventionnaliste que se fait Buffon des vérités mathématiques, lesquelles «se réduisent toutes à des vérités de définition» $(\S \mathrm{II})^{30}$. Les mathématiques ne peuvent donc nous renseigner sur la structure du réel, mais seulement intervenir comme instruments de mise en ordre de nos connaissances, grâce à l'introduction de la mesure. Ceci est particulièrement vrai de l'arithmétique. Le nombre est pensé par Buffon comme un instrument de mesure ; il n'est, écrit-il au §XXIV, «qu'un assemblage d'unités de même espèce». Or le nombre est une abstraction artificielle, n'ayant pas d'existence indépendante par rapport aux réalités qu'il permet de nombrer. Il convient donc de limiter l'arbitraire des définitions ${ }^{31}$ en les appropriant à leurs applications, c'est-à-dire aux nécessités de la vie individuelle et de la vie sociale, par conséquent de corriger, en quelque sorte, l'abstraction mathématique par la considération du point de vue moral.

D'un autre côté, l'estimation des risques ou celle de la valeur des biens échangés sont abandonnées à l'arbitraire du sentiment individuel ou d'unités de mesures particulières et hétérogènes, arbitraire qui rend possible, précise Buffon, le développement de l'escroquerie.

Le projet de Buffon est alors de substituer à ces mesures arbitraires et variables une mesure à la fois universelle et moralement fondée. C'est notamment l'objectif que

\footnotetext{
${ }^{28}$ Ainsi, le mémoire «Solution de problèmes sur le jeu du franc-carreau» présenté à l'Académie Royale des Sciences en 1733 est repris au § XXIII, la Préface à la traduction de La Méthode des fluxions de Newton publiée en 1740 est reprise au $\S$ XXIV.

${ }^{29}$ En effet, une note du $\S$ VIII cite une lettre de Daniel Bernoulli datée de mars 1762 contestant son estimation de la certitude morale. De plus, le $\S$ XVII fait référence, à propos du problème de SaintPétersbourg, aux Mémoires mathématiques de Fontaine des Bertins publiés en 1764. On peut ajouter que l'analyse de la «valeur morale» de l'espérance est au moins antérieure à 1767, puisque d'Alembert y fait allusion dans le mémoire «Sur l'analyse des jeux», (p. 79), publié en 1768 dans le tome IV de ses Opuscules mathématiques, et dont on sait qu'il fut écrit au plus tard en 1767, comme l'atteste, ainsi que me l'a précisé Pierre Crépel, la correspondance de Lagrange.

${ }^{30} \mathrm{Cf}$. également «De la manière d'étudier et de traiter l'histoire naturelle», in Histoire naturelle, générale et particulière, Paris, Imprimerie royale, tome premier, 1749, p. 53 : «Il y a plusieurs espèces de vérités, et on a coutume de mettre dans le premier ordre les vérités mathématiques, ce ne sont cependant que des vérités de définitions ; ces définitions portent sur des suppositions simples, mais abstraites, et toutes les vérités en ce genre ne sont que des conséquences composées, mais toujours abstraites, de ces définitions».

${ }^{31}$ «Comme les définitions sont les seuls principes sur lesquels tout est établi, et qu'elles sont arbitraires et relatives, toutes les conséquences qu'on en peut tirer sont également arbitraires et relatives», ibid., p. 54.
} 
poursuit le développement consacré aux mesures arithmétiques et aux mesures géométriques.

Ainsi, le passage sur les mesures arithmétiques a sans doute pour objet, comme c'était le cas du mémoire dont il est issu ${ }^{32}$, de fournir une règle générale permettant de «transporter un nombre d'une échelle d'arithmétique dans une autre», mais le but de Buffon n'est pas ici purement spéculatif. Il entend proposer une réforme du système de numération, à savoir substituer le système duodécimal au système décimal, afin de réduire l'imperfection inhérente à la mesure. Buffon s'en explique par deux raisons. La première est que le même nombre, exprimé en système duodécimal, a besoin de moins de caractères pour être transcrit que s'il est exprimé en système décimal ${ }^{33}$, donc est d'un usage plus commode. La seconde raison, plus déterminante, est que le système duodécimal a plus de diviseur que le système décimal $(2,3,4$ et 6 contre 2 et 5$)$, donc permet d'obtenir plus fréquemment une mesure entière lorsqu'il s'agit de subdiviser une mesure initiale en sous-unités. Il est donc mieux approprié aux «besoins de la vie civile», comme en témoigne le fait, signale Buffon, qu'il est réellement appliqué concurremment au système décimal, non seulement pour les mesures de temps, mais aussi dans l'activité commerciale où l'unité est souvent la douzaine ou la grosse (douzaine de douzaine), et dans la mesure des distances, puisque, précise Buffon, «le pied est dans l'échelle duodénaire la troisième puissance de la ligne, le pouce la seconde puissance» ( $§$ XXVII). Buffon propose ainsi une réforme visant à simplifier et uniformiser l'opération de mesure nécessaire à la pratique commerciale, même s'il ne s'étend pas sur les conséquences économiques de cette réforme.

De même, le développement sur les mesures géométriques insiste sur la nécessité de substituer une unité universelle et invariable, dont Buffon aperçoit l'étalon dans la longueur du pendule qui bat la seconde sous l'équateur ${ }^{34}$, aux unités de mesures en usage, arbitraires parce qu'héritées de circonstances historiques et politiques, et favorisant du même coup la malhonnêteté dans les transactions ${ }^{35}$, malhonnêteté croissant, précise Buffon, avec la richesse nationale ${ }^{36}$. Son analyse remplit ainsi une fonction critique et curative, en ce qu'elle vise à dénoncer et supprimer «l'incertitude et les embarras du commerce».

\footnotetext{
${ }^{32}$ Buffon, «Formule sur les échelles arithmétiques», in Mémoires de l'Académie Royale des Sciences de Paris, 1744 pour l'année 1741, pp. 219-222.

${ }^{33}$ Par exemple, tandis que le nombre 100 nécessite en base 10 trois caractères $(1,0,0)$, il en suffit de deux $(8,4)$ pour l'exprimer dans le système duodécimal.

${ }^{34}$ Si l'universalité de l'unité de mesure géométrique lui est assurée par son enracinement physique, il n'en va pas de même pour les mesures arithmétiques, car c'est justement à raison de son origine naturelle que Buffon dénonçait l'arbitraire du système décimal, dont l'adoption généralisée résulte, selon lui, de la conformation de la main, l'homme comptant d'abord sur ses doigts. Cette inversion de la relation à la naturalité tient à ce que pour Buffon la mesure arithmétique est purement intellectuelle, tandis que la géométrie est plus appropriée à la mesure du réel.

${ }^{35}$ «Chaque peuple a fait et adopté ses mesures, chaque État, chaque Province a les siennes ; l'intérêt et la mauvaise foi dans la société ont dû les multiplier», écrit Buffon au $§$ XXX.

${ }^{36}$ «Chez des peuples plus dénués d'arts, et moins éclairés pour leurs intérêts que nous ne le sommes, la multiplication des mesures n'aurait peut-être pas eu d'aussi mauvais effets...; mais plus un terrain est précieux, plus une denrée est chère, plus aussi les mesures sont épluchées \& contestées, plus on met d'art \& de combinaison dans les abus qu'on en fait ; la fraude est allée jusqu'à imaginer plusieurs mesures difficiles à comparer, elle a su se couvrir en mettant en avant ces embarras de convention», ibid.
} 
C'est ce même objectif, fonder la mesure sur une unité universelle et pratiquement appropriée, qui est à l'œuvre dans l'analyse des valeurs morales de la certitude et de l'espérance.

En effet, la mesure de la certitude morale et la prise en compte de la valeur morale de l'espérance doivent permettre d'estimer la «valeur réelle» de nos risques, grâce à laquelle il est possible de dénoncer la fraude et la malhonnêteté à l'œuvre dans les jeux de hasard proprement dit, et plus largement de régler toute forme de convention où intervient le risque. Buffon assigne ainsi une fonction critique et thérapeutique à son analyse, dont le but est d'accorder l'opération de mesure aux nécessités de la pratique, ou comme il le dit à propos du problème de Saint-Pétersbourg, de lever «la contrariété du bon sens et du calcul» (§ XVI) ; cet accord passant par la détermination du seuil à partir duquel une probabilité peut être considérée comme pratiquement négligeable, seuil calculé en référence à la probabilité de mort subite pour un homme moyen que rien n'expose particulièrement au risque de mort, et qui est susceptible, à ce titre, d'avoir valeur générale ${ }^{37}$.

(Il faut reconnaître cependant que, si l'orientation de l'analyse est ici semblable à celle que manifeste le développement sur les mesures arithmétiques et géométriques, son objet est différent, car l'analyse ne concerne plus ici une base de numération ou une unité de mesure, mais bien une mesure, et la recherche d'une détermination générale de la valeur de la certitude morale, donc aussi d'une mesure des probabilités pratiquement négligeables valant universellement, peut apparaître comme une abstraction, sans efficace pratique lorsqu'il s'agira de l'appliquer effectivement, donc de la confronter à des problèmes réels toujours particuliers, ce que ne manquera pas de faire observer Condorcet ${ }^{38}$ ).

En définitive, l'Essai d'arithmétique morale présente non pas une réflexion s'inscrivant directement dans le champ de l'arithmétique politique, mais un ensemble d'éléments susceptibles de s'y appliquer, soit parce qu'ils permettent d'éclairer nos décisions dans l'incertain, soit par les conséquences économiques et sociales qu'ils impliquent. Cependant, cette application, n'est pas effectuée par Buffon, dont la réflexion, lorsqu'elle quitte la sphère de l'abstraction, prend moins pour objet les phénomènes politiques ou économiques eux-mêmes que les conséquences sociales de comportements individuels. L'Essai d'arithmétique morale peut, en ce sens, apparaître comme un discours préliminaire à l'arithmétique politique, ce qu'attesterait sa situation éditoriale, puisqu'il précède le vaste exposé des Probabilités de durée de la vie et des articles démographiques qui les suivent.

\section{LES PROBABILITÉS DE LA DURÉE DE LA VIE}

Ce texte nous place-t-il enfin sur le terrain de l'arithmétique politique ? Avant de répondre à cette question, il convient déjà d'en indiquer la date probable de composition et le contenu.

\footnotetext{
${ }^{37}$ Constatant que cette probabilité est, pour un homme moyen âgé de 56 ans, de 1/10189, Buffon en conclut que «toute probabilité égale ou plus petite, doit être regardée comme nulle, et que toute crainte ou toute espérance qui se trouve au-dessous de dix mille, ne doit ni nous affecter ni même nous occuper un seul instant le cœur ou la tête», (§ VIII). Sur l'argumentation déployée par Buffon pour fournir cette estimation de la certitude morale, on peut trouver quelques indications dans T. Martin, «Certitude et probabilité selon Buffon», L'Enseignement philosophique, 49e année, n 3, jan.-fév. 1999, pp. 5-18.

${ }^{38}$ Cf. «Éloge de M. le Comte de Buffon», in Histoire de l'Académie Royale des Sciences, 1788, cité dans Condorcet, Arithmétique politique. Textes rares ou inédits (1767-1789), édité par B. Bru et P. Crépel, Paris, I.N.E.D., 1994, p. 656.
} 
Le texte date vraisemblablement des années 1760 . Il est bien sûr postérieur à 1749 , et sans doute antérieur à 1762, puisque le $\S$ VIII de l'Essai d'arithmétique morale qui renvoie à ce texte pour la mesure de la probabilité de mort subite d'un homme de 56 ans cite, comme on l'a dit (cf. supra note 29), une lettre de Daniel Bernoulli de mars 1762. De plus, Buffon fait référence, à propos de la faute, d'impression relative au nombre de morts pour la $4^{\mathrm{e}}$ année (12477 au lieu de 13477), au tome II des Opuscules mathématiques de d'Alembert publié en 1761, et au tome V des Mélanges de littérature, d'histoire et de philosophie publié en 1767. On peut donc supposer que le texte était pour l'essentiel composé avant 1762, et fut revu après 1767.

Cet exposé est explicitement placé par Buffon dans le prolongement de l'analyse de 1749, puisqu'il précise que ces probabilités de durée de vie peuvent être calculées à partir de la table de mortalité qu'il a publiée, sans d'ailleurs rappeler qu'elle est due à Dupré de Saint-Maur. Tout se passe donc comme si, aux yeux de Buffon, l'étude de la mortalité humaine n'avait en rien progressé depuis 1749, illustrant par là le jugement que Cournot portera sur lui, affirmant qu'il «ne croyait à rien qu'à ses propres idées» ${ }^{39}$.

Le texte de 1777 manifeste cependant deux perfectionnements par rapport à celui de 1749.

D'une part, Buffon ne s'en tient pas aux données brutes fournies par la table de mortalité, mais procède à un ajustement visant à corriger l'excès dont bénéficient les âges correspondant à des nombres ronds $(5,10,15,20,30$, etc.). Ceux-ci, en effet, comme il le notait déjà en 1749 mais sans se préoccuper alors d'y remédier, présentent une mortalité anormalement élevée par rapport aux autres âges, due au fait que les curés, surtout dans les campagnes, ne notent pas l'âge exact du défunt, mais l'arrondissent souvent à la dizaine demi-dizaine inférieure ou supérieure, et ceci d'autant plus facilement, note Buffon, que les paysans ne connaissent leur âge qu'à un ou deux ans près. La méthode de correction qu'il utilise (cf. annexe 4) consiste, à partir de la $10^{\mathrm{e}}$ année, à ordonner la série des nombres de morts par année en groupes de 5 années consécutives, en calculer la moyenne, puis répartir autour de cette moyenne les 4 autres nombres de telle sorte que :

$\left.1^{\mathrm{e}}\right)$ leur somme demeure inchangée,

$2^{\text {e) }}$ ils aillent en augmentant ou diminuant selon que la somme des nombres du groupe suivant est supérieure ou inférieure à celle des nombres du groupe considéré. La correction des données brutes permet ainsi de restituer à la série sa continuité, accidentellement compromise par des irrégularités circonstancielles. Buffon reconnaît ainsi implicitement, comme le note Maurice Fréchet, que «des nombres artificiellement construits [sont] plus «vrais» que les nombres résultant directement de l'observation» ${ }^{40}$, ce qui est certes une avancée par rapport au texte de 1749, mais non une innovation de Buffon, puisque Deparcieux procédait déjà à une semblable correction dans son Addition à l'Essai sur les probabilités de la durée de la vie humaine de 1760 (cf. annexe 5).

Cependant, si la règle formulée par Buffon est claire, son application pose problème, car les chiffres de la table corrigée ne correspondent pas à ceux qu'il annonce (cf. colonne $\mathrm{K}$ du tableau de l'annexe 3). Ainsi, la somme des décès figurant dans la table corrigée pour les $10^{\mathrm{e}}$ à $14^{\mathrm{e}}$ années est 477 , et non 441 . De plus, Buffon justifiait la nécessité de l'ajustement en précisant que le nombre de décès pour la $10^{\mathrm{e}}$ année, soit 114 , est trop fort. Or, il lui substitue 112, nombre dont on ne peut pas dire qu'il s'écarte nettement de 114.

\footnotetext{
${ }^{39}$ Cournot, Considérations sur la marche des idées et des événements dans les temps modernes, Paris, 1872, 1. IV, ch. 1 ; rééd. Paris, Vrin, 1973, p. 279.

${ }^{40}$ Maurice Fréchet, article cité supra à la note 2, p. 436.
} 
Deux indices, cependant, permettent d'affirmer qu'il ne s'agit pas là d'une erreur de la part de Buffon, mais d'une complication implicite de la méthode ${ }^{41}$. En effet, si l'on considère non plus les décès des $10^{\mathrm{e}}$ à $14^{\mathrm{e}}$ années, mais qu'on leur ajoute ceux des 5 années suivantes, on retrouve l'égalité de leurs sommes calculées pour 1749 et pour 1777. De plus, il faut avouer que les chiffres proposés par Buffon en 1777 permettent effectivement de restituer à la série sa continuité. Notre hypothèse est plutôt que Buffon, après avoir appliqué sa méthode de correction, a repris la série ainsi obtenue pour la modifier afin d'en accentuer la continuité, mais en omettant de préciser quelle nouvelle opération il avait effectuée.

D'autre part, il ne se contente plus, comme il le faisait en 1749 , de mesurer la vie probable, qu'il appelle ici «vie moyenne». Il effectue un calcul beaucoup plus développé, visant à déterminer, pour chacun des âges compris entre la naissance et 100 ans, la probabilité de vivre $n$ années ( $n$ étant pris tous les 5 ans à partir de l'âge de 2 ans). Ce calcul consiste à rapporter pour l'âge $x$, le nombre de survivants à l'âge $x+n$ au nombre de survivants à l'âge $x^{42}$ (cf. annexe 6).

Si Buffon ne s'explique pas sur les motifs qui président à ce changement de méthode, on peut légitimement supposer qu'il résulte des débats qui animèrent l'Académie des sciences sur cette question dans les années 1760.

La question se pose de savoir quel but poursuit Buffon en publiant cette longue et fastidieuse suite de calculs répétitifs, s'étendant sur plus de 120 pages, car outre l'introduction méthodologique et un court commentaire sur la mortalité en général, inséré après les probabilités de durée de vie d'un nouveau-né et d'un enfant d'un an, l'ensemble du texte se réduit à l'énumération des résultats du calcul. Notamment, il ne se propose ni de calculer des rentes viagères, auxquelles il ne fait allusion qu'incidemment ${ }^{43}$, ni, ce qui peut paraître plus étonnant, d'éclairer la question de l'inoculation de la petite vérole, dont il ne souffle mot. En réalité, son objectif est essentiellement, comme il l'était en 1749, de fournir un ensemble de vérités générales sur la mortalité de l'espèce humaine. Ainsi Buffon indique, par exemple, que «les deux tiers du genre humain périssent avant l'âge de trente-neuf ans, en sorte qu'il n'y a guère qu'un tiers des hommes qui puisse propager l'espèce» ${ }^{44}$, ou encore que «les trois quarts du genre humain périssent avant l'âge de 51 ans, c'est-à-dire avant d'avoir rien achevé pour soi-même, peu fait pour sa famille, et rien pour les autres» ${ }^{45}$. Son analyse lui permet notamment d'établir la thèse générale selon

\footnotetext{
${ }^{41}$ La consultation de l' Addition à l'Essai sur les probabilités de la durée de la vie humaine de Deparcieux n'est ici d'aucun secours, car la table corrigée qu'il présente aux pages 34-35 de l'ouvrage ne manifeste pas de discordance entre la méthode annoncée et sa mise en œuvre. Ainsi, pour reprendre son exemple (cf. annexe 5), la série des décès pour les $48^{\mathrm{e}}$ à $52^{\mathrm{e}}$ années, soit 32, 46, 159, 63, 44 devient après correction : 70, 70, 69, 68, 67.

${ }^{42}$ Par exemple, dit Buffon, pour une personne de 30 ans, on peut parier 8619 contre 776 qu'elle vivra 5 ans de plus, 8619 représentant le nombre de survivants 5 ans après la fin de la trentième année, et 776 la somme des morts entre la fin de la $30^{\mathrm{e}}$ année et la fin de la $35^{\mathrm{e}}$, soit la différence entre le nombre de survivants entrés dans leur $31^{\mathrm{e}}$ année et le nombre de survivants entrés dans leur $36^{\mathrm{e}}$ année.

43 «Une rente viagère sur la tête d'un enfant d'un an», écrit Buffon à la page 169 , «vaut le double d'une rente viagère sur une personne de quarante-huit ans, et le triple de celle que l'on placerait sur la tête d'une personne de soixante ans. Tout père de famille qui veut placer de l'argent à fonds perdu doit préférer de le mettre sur la tête de son enfant d'un an, plutôt que sur la sienne s'il est âgé de plus de vingt-un ans».

${ }^{44}$ Histoire naturelle, générale et particulière, servant de suite à l'Histoire naturelle de l'Homme, Supplément, tome quatrième, Paris, Imprimerie Royale, 1777, p. 161.

${ }^{45}$ Ibid., p. 162.
} 
laquelle «une raison pour vivre est d'avoir vécu» ${ }^{46}$, puisque la mortalité la plus élevée étant la mortalité infantile, plus on s'éloigne de l'enfance, plus l'espérance de vie augmente, et puisque celle-ci décroît ensuite d'autant moins vite que la durée de vie s'allonge, si bien que, dit-il, «plus la mort s'approche et plus sa marche se ralentit ${ }^{47}$.

En revanche, il ne se préoccupe nullement de développer les applications économiques ou sociales que rendent possibles les informations délivrées par les tables de mortalité et les probabilités qu'elles permettent de calculer, ni de justifier la méthode de calcul qu'il met en œuvre, puisque sa référence aux écrits de d'Alembert ${ }^{48}$ vise seulement à dénoncer l'erreur que celui-ci aurait commise dans la détermination de la probabilité de durée de vie des nouveau-nés, erreur (faussement) attribuée à la faute d'impression que comportait l'édition du texte de 1749 , et non la discussion méthodologique à laquelle il soumet les calculs de probabilité de durée de vie aux tomes II et IV de ses Opuscules mathématiques ${ }^{49}$.

\section{LES ÉTUDES DÉMOGRAPHIQUES DE 1777}

L'exposé des Probabilités de la durée de la vie est suivi d'un ensemble d'études qui tranchent avec le style de l'analyse précédente. Il s'agit de onze études, plus tardives, puisque composées entre $1767^{50}$ et, au plus tôt, 1775 , présentées sans lien avec ce qui précède, et portant sur la natalité, la fécondité et la mortalité à Paris, à Londres et dans diverses localités bourguignonnes - nous en donnons la liste à l'annexe 7 -, où Buffon entreprend de dégager, par la comparaison entre les différentes tables mobilisées, des faits dominants, expressifs de la stabilité ou au contraire de la variation des paramètres démographiques selon le sexe, le lieu, voire la situation sociale. Il fait donc ici davantage œuvre de démographe, ou plutôt il applique aux registres des paroisses la méthode même qu'il préconise en histoire naturelle. Il convient, précisait-il en effet dans le $1^{\mathrm{er}}$ discours de l'Histoire naturelle, de partir d'abord de la description des faits particuliers, pour ensuite «combiner les observations, généraliser les faits, les lier ensemble par la force des analogies, et tâcher d'arriver à ce haut degré de connaissance où nous pouvons juger que les effets particuliers dépendent d'effets plus généraux ${ }^{51}$.

Sans se livrer à une analyse détaillée des différents résultats auxquels parvient Buffon, analyse déjà effectuée par Lucien Badet et J. Dupâquier ${ }^{52}$, on peut, pour en saisir l'esprit général, s'efforcer d'en mettre à jour les traits principaux, en en retenant trois :

- Le premier caractère que l'on peut relever est l'importance relative accordée par Buffon aux facteurs d'origine physique, et plus directement climatiques, dans

\footnotetext{
${ }^{46}$ Ibid., p. 163.

${ }^{47}$ Ibid., p. 163. Ainsi, écrit Buffon, «un homme de quatre-vingts ans, qui vit un an de plus, gagne sur elle cette année presque tout entière, puisque de quatre-vingts à quatre-vingt-un ans, il ne perd que deux mois d'espérance de vie sur trois ans et sept mois», ibid., p. 164.

${ }^{48}$ Ibid., p. 161.

${ }^{49} \mathrm{Cf}$. D'Alembert, «Note sur le mémoire Sur l'application du calcul des probabilités à l'inoculation de la petite vérole» (11 ${ }^{\mathrm{e}}$ mémoire), in Opuscules mathématiques, tome II, 1761, pp. 74-76 et «Sur la durée de la vie», in Opuscules mathématiques, tome IV, 1768, pp. 92-98.

${ }^{50}$ Dans une note de l'article consacré à la population parisienne, visant à signaler le nombre de décès pour l'année 1772, relevé dans la Gazette du 22 janvier 1773, Buffon précise que l'article lui-même fut écrit en 1767.

${ }^{51}$ «De la manière d'étudier et de traiter l'histoire naturelle», in Histoire naturelle..., tome I, 1749, p. 51.

${ }^{52}$ Cf. supra, notes 2 et 3 .
} 
l'explication des phénomènes démographiques, importance qui se lit dans le fait que les explications économiques ou sociales sont généralement doublées par une explication de type physique. Ainsi, Buffon reconnaît le rôle des facteurs économiques et sociaux, lorsque, par exemple, il aperçoit une relation de dépendance entre abondance et fécondité, et corrélativement entre disette et stérilité, mais il l'accompagne du constat de la variabilité de la fécondité selon les saisons, et il étend cette dernière remarque à la mortalité, indiquant que «c'est après l'hiver, et au commencement de la nouvelle saison, que les hommes, comme les plantes, périssent en grand nombre» ${ }^{53}$. De même, de l'analyse comparée des mortalités française et anglaise, il retient principalement que «la salubrité de l'air en France est plus grande qu'en Angleterre». C'est d'ailleurs encore en référence à cette qualité de l'air qu'il conteste le multiplicateur 30 adopté notamment par Graunt, Petty, et Corbyn-Morris pour calculer la population de Londres à partir de sa mortalité moyenne, car adopter ce nombre reviendrait pour Buffon, qui estime ce multiplicateur à 35, à supposer que la différence de salubrité de l'air entre Paris et Londres est dans un rapport de 35 à 30, rapport vraisemblablement excessif ${ }^{54}$. De même encore, pour s'efforcer de rendre compte de la supériorité des naissances féminines par rapport aux naissances masculines dans le bailliage de Semur-en-Auxois, Buffon invoque deux «conjectures raisonnables» ${ }^{55}$, à savoir qu'il naît davantage de filles dans les régions économiquement moins favorisées, mais aussi, semble-t-il, dans les montagnes plutôt que dans les vallées.

- Le second trait marquant que l'on peut relever, même s'il n'est pas original, est le contraste entre le souci d'exactitude manifesté par Buffon dans la restitution des données statistiques et la hardiesse des généralisations qu'il en induit. Ainsi, pour estimer la population du royaume, il considère la mortalité moyenne dans les 138 paroisses des bailliages de Semur-en-Auxois pour les années 1770-1774 et Saulieu pour les années 1770-1772, et la multiplie par le coefficient 35. Empruntant à Expilly l'affirmation selon laquelle la France compte 41000 paroisses, il estime alors que «la population dans ces deux bailliages de Semur et de Saulieu est à la population de tout le royaume à très peu près comme 138 sont à $41000{ }^{56}$, supposant ainsi d'avance résolue la question de savoir si l'on peut étendre à la France entière ce qu'il a constaté pour les deux bailliages bourguignons. Et ce choix du multiplicateur 35, également utilisé pour estimer la population parisienne, n'est lui-même justifié que par l'analyse des dénombrements des populations de Montbard, Semur et Époisses.

- Enfin, il est possible de soupçonner la présence chez Buffon d'une préoccupation idéologique venant se superposer à l'étude proprement scientifique des données. En effet, l'analyse des tables pour Paris et la comparaison entre la mortalité parisienne et la mortalité dans les campagnes visent principalement à souligner l'amélioration des conditions de vie dont bénéficieraient les habitants de la capitale, attestée notamment par le fait que :

$1^{\circ}$ ) la croissance de la population de Paris n'est pas proportionnelle à son extension en superficie, si bien que «la moitié de cette extension, estime Buffon, ne tient qu'à la «commodité», et non à la nécessité» ${ }^{57}$,

\footnotetext{
${ }^{53}$ Cf. «État des baptêmes, mariages et morts pour Paris et faubourgs», Histoire naturelle..., Supplément, tome IV, 1777 , p. 282.

${ }^{54}$ Histoire naturelle..., Supplément, tome IV, p. 317.

${ }^{55}$ Ibid., p. 298.

${ }^{56}$ Ibid., p. 302. Buffon estime la population du royaume à un total de 21672777 habitants, chiffre proche, note-t-il, de celui calculé par Expilly, soit 22014357.

${ }^{57}$ Ibid., p. 281.
} 
$2^{\circ}$ ) la fécondité de la population parisienne suffit presque entièrement à son renouvellement,

$3^{\circ}$ ) la longévité moyenne est plus grande à Paris que dans les campagnes. En regard, la comparaison de la mortalité à Londres et à Paris permet à Buffon d'établir que «Londres a besoin de se recruter de plus de moitié du nombre de ses naissances pour s'entretenir, tandis que Paris se suffit à lui-même à un soixante-quinzième près ${ }^{58}$, cette nécessité d'un supplément extérieur de population allant certes en s'affaiblissant, mais non du fait d'une amélioration des conditions de vie à Londres, mais en raison de la diminution de la population londonienne. Bref, il s'agit ici pour Buffon de prendre position dans le déjà classique $^{59}$ débat démographique, mais aussi politique, sur la prospérité respective de Londres et de Paris, et plus largement de la France et de l'Angleterre.

\section{CONCLUSION}

Finalement, lorsqu'on veut saisir globalement les travaux de Buffon susceptibles d'intéresser l'arithmétique politique pour en dégager l'unité ou un projet d'ensemble, on se heurte à leur hétérogénéité et à leur morcellement. On peut cependant en indiquer l'orientation générale. Buffon, dans son Histoire naturelle de l'homme, insiste sur l'unité de l'humanité, envisagée d'un double point de vue : physique, comme espèce déterminée physiologiquement ; moral, en tant qu'elle rassemble des individus qui partagent le fait d'être tous différents, parce que doués de conscience et de volonté. L'humanité est ainsi pensée dans son unité au niveau de l'espèce, dans sa diversité à celui de l'individu ; ce qui laisse peu de place pour reconnaître une spécificité au champ socio-politique. L'homme existe d'abord à la fois comme être biologique et comme individu raisonnable et prévoyant, secondairement comme être social ; le corps social étant, pour Buffon, comme le corps organique, «un tout qui s'explique par la composition de ses parties», ainsi que le remarquait Canguilhem ${ }^{60}$. Il n'est donc pas étonnant que Buffon ne fasse guère porter son attention sur la diversité possible des formes d'organisation et d'administration collectives, qu'elles soient économiques, sociales ou politiques. D'un autre côté, il se préoccupe moins de développer les applications des mathématiques aux questions socio-politiques, que d'en interroger la fécondité et les limites. En un mot, lorsqu'il appréhende les phénomènes sociaux, Buffon est plus moraliste que politique et plus philosophe que mathématicien.

On comprend mieux alors le contraste qui caractérise les jugements portés sur cette partie de son œuvre. Les non-démographes (Lucien Badet, Maurice Fréchet) découvrent un Buffon qu'ils ne s'attendaient pas à rencontrer; les historiens de la démographie (Jacques Dupâquier, Lazare Behar) ne peuvent qu'être déçus de ne pas y trouver des développements qu'en réalité, ils ne pouvaient attendre eu égard au projet qui est le sien.

\footnotetext{
${ }^{58}$ Ibid., p. 317.

${ }^{59}$ Déjà classique puisqu'il est engagé dès le milieu du XVII ${ }^{e}$ siècle, cf. J. Dupâquier, «Londres ou Paris ? Un grand débat dans le petit monde des arithméticiens politiques (1662-1759)», Population, janvier-avril 1998, n 1-2, pp. 311-325. Le débat ne s'achève d'ailleurs pas en 1759, puisqu'on en trouve l'écho non seulement chez Buffon, mais aussi, par exemple, chez Arthur Young, cf. Arithmétique politique, trad. M. Freville, La Haye, 1775, vol. 1, ch. IX.

${ }^{60}$ G. Canguilhem, La connaissance de la vie, Paris, Hachette, $1952 ; 2^{\mathrm{e}}$ édition, Vrin, 1975, p. 57. «Si Buffon distingue nettement la société humaine d'une simple agrégation mécanique d'éléments primitifs, il demeure que la vie du corps social est le fruit des individus qui le composent, comme celle de l'organisme résulte des «molécules organiques» qui le constituent».
} 
Cependant, sur la base de cette orientation générale, se dessine une évolution de la relation de Buffon à l'arithmétique politique, dont témoigne la série des textes du volume de 1777, que l'on peut ordonner selon la progression suivante :

$1^{\circ}$ ) Une réflexion sur la mesure, qui accorde une place non négligeable au calcul des probabilités,

$2^{\circ}$ ) L'application de ce calcul, à titre d'instrument de mesure, à la mortalité en général,

$3^{\circ}$ ) Enfin, plus tardivement (trop tardivement d'ailleurs selon les historiens de la démographie $^{61}$ ), un ensemble d'études qui ne s'en tiennent plus à une perspective générale, mais prennent également en compte le particulier.

Or, cette progression menant du général vers le particulier ne se réduit pas à un ordre d'exposition, puisque d'une part ce particulier ne vient pas illustrer la réflexion générale initiale, ces textes étant, comme on l'a vu, laissés dans une indépendance réciproque, et de l'autre, elle coïncide, semble-t-il, avec l'ordre chronologique d'élaboration des textes ${ }^{62}$. Si l'on accepte cette hypothèse, il faut alors supposer qu'il y aurait, dans la pensée de Buffon, un intérêt croissant, quoique là encore tardif, pour les applications du calcul au domaine social.

Il reste que ces textes soulèvent plusieurs questions non résolues. Elles concernent, on l'a vu, les méthodes de calcul mises en œuvre par Buffon, mais on peut en ajouter ici une autre, de nature historique, tenant à leur date de publication. Les deux premiers tomes du Supplément à l'Histoire naturelle, publiés respectivement en 1774 et 1775, servent de suite à la Théorie de la terre, qui ouvrait l'Histoire naturelle en son tome premier. On pourrait donc s'attendre à ce que, conformément au plan de l'Histoire naturelle, le tome III du Supplément vienne compléter l'Histoire naturelle de l'homme. Or, ce tome III, publié en 1776, fait suite à l'Histoire des animaux quadrupèdes, le supplément à l'Histoire naturelle de l'homme n'intervenant qu'avec le tome IV. Il y a là une inversion, comme si Buffon avait retardé la publication de ses écrits d'arithmétique morale et politique d'une année, dont nous ignorons si elle tient à la préparation du texte ou à d'autres circonstances.

\footnotetext{
${ }^{61}$ cf. J. et M. Dupâquier, Histoire de la démographie, Paris, Librairie académique Perrin, 1985, p. 174 : «l'Essai d'arithmétique morale [auquel ces auteurs intègrent les études démographiques] arrive trop tard: en 1777, les travaux d'Expilly et de Messance sont déjà publiés ; du point de vue de la méthode et des problèmes soulevés, ils apparaissent bien supérieurs à ce qu'apporte alors Buffon».

${ }^{62} \mathrm{Si}$ le $§$ XXVII de l'Essai d'arithmétique morale concernant les systèmes de numération reprend le mémoire «Formule sur les échelles arithmétiques» qui date vraisemblablement de 1738 - il est publié dans les Mémoires de l'Académie Royale des Sciences de Paris de 1744, mais mention y est faite dans les Registres de l'Académie les 23 décembre 1738 et 25 février 1739 -, ce dernier s'en tient à la technique mathématique, à l'exclusion de toute préoccupation économique ou sociale. Pour ce qui concerne notre objet, et si nos remarques historiques précédentes sont exactes, le premier texte de la série daterait des années 1760-1764, le second, antérieur à 1762, serait modifié après 1767, le troisième enfin serait pour l'essentiel postérieur à 1775 .
} 


\section{ANNEXE 1}

De la vieillesse et de la mort, in Histoire naturelle..., tome I, 1749, p. 590.

\begin{tabular}{|c|c|c|c|c|c|c|c|c|c|c|c|}
\hline \multirow[t]{2}{*}{ PAROISSES } & \multirow[t]{2}{*}{ MORTS } & \multicolumn{5}{|c|}{ Années de la vie } & \multicolumn{5}{|c|}{ Années de la vie } \\
\hline & & 1 & 2 & 3 & 4 & 5 & 6 & 7 & 8 & 9 & 10 \\
\hline Clermont......... & 1391 & 578 & 73 & 36 & 29 & 16 & 16 & 14 & 10 & 8 & 4 \\
\hline Brinon............ & 1141 & 441 & 75 & 31 & 27 & 10 & 16 & 9 & 9 & 8 & 5 \\
\hline Jouy............... & 588 & 231 & 43 & 11 & 13 & 5 & 8 & 4 & 6 & 1 & 0 \\
\hline Lestiou........... & 223 & 89 & 16 & 9 & 7 & 1 & 4 & 3 & 1 & 1 & 1 \\
\hline Vandeuvre......... & 672 & 156 & 58 & 18 & 19 & 10 & 11 & 8 & 10 & 3 & 2 \\
\hline Saint-Agil........... & 954 & 359 & 64 & 30 & 21 & 20 & 11 & 4 & 7 & 2 & 7 \\
\hline Thury............. & 262 & 103 & 31 & 8 & 4 & 3 & 2 & 2 & 2 & 1 & 2 \\
\hline Saint-Aman......... & 748 & 170 & 61 & 24 & 11 & 12 & 15 & 3 & 6 & 8 & 6 \\
\hline Montigny......... & 833 & 346 & 57 & 19 & 25 & 16 & 21 & 9 & 7 & 5 & 5 \\
\hline Villeneuve....... & 131 & 14 & 3 & 5 & 1 & 1 & 0 & 0 & 0 & 0 & 0 \\
\hline Goussainville... & 1615 & 565 & 184 & 63 & 38 & 34 & 21 & 17 & 15 & 12 & 8 \\
\hline Ivry............. & 2247 & 686 & 298 & 96 & 61 & 50 & 29 & 34 & 26 & 13 & 19 \\
\hline \multicolumn{12}{|c|}{ Total des Morts $\quad 10805$} \\
\hline \multicolumn{3}{|c|}{ 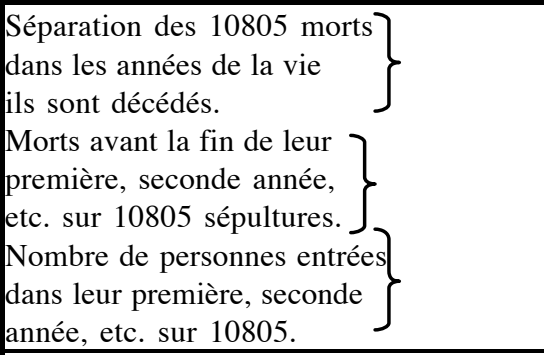 } & 4701 & 5051 & 5754 & 5498 & 5639 & 5166 & 5059 & 4960 & 5966 \\
\hline Saint-André......... & 1728 & 201 & 122 & 94 & 82 & 50 & 35 & 28 & 14 & 8 & 7 \\
\hline Saint-Hippolite.... & 2516 & 754 & 361 & 127 & 64 & 60 & 55 & 25 & 16 & 20 & 8 \\
\hline Saint-Nicolas...... & 8945 & 1701 & 962 & 414 & 298 & 221 & 162 & 147 & 111 & 64 & 40 \\
\hline Total des Morts & 13189 & & & & & & & & & & \\
\hline \multirow{3}{*}{\multicolumn{2}{|c|}{ 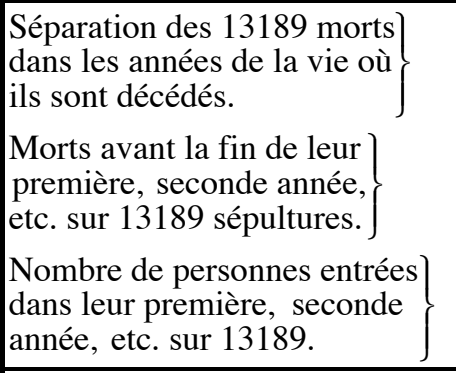 }} & 2716 & 1415 & 635 & 444 & 331 & 252 & 200 & 141 & 92 & 55 \\
\hline & & 2716 & 4131 & 4766 & 5210 & 5541 & 5793 & 5993 & 6134 & 6226 & 6281 \\
\hline & & 13189 & 10473 & 9058 & 8423 & 7979 & 7648 & 7396 & 7196 & 7055 & 6963 \\
\hline \multicolumn{2}{|c|}{$\begin{array}{l}\text { Séparation des } 23994 \text { morts: } \\
\text { dans les années de la vie où } \\
\text { ils sont décédés. }\end{array}$} & 6454 & 2378 & 985 & 700 & 509 & 406 & 307 & 240 & 154 & 114 \\
\hline \multicolumn{2}{|c|}{$\begin{array}{l}\text { Morts avant la fin de leur } \\
\text { première, seconde année, } \\
\text { etc. sur } 23994 \text { sépultures. }\end{array}$} & 6454 & 8832 & 9817 & 10517 & 11026 & 11432 & 11739 & 11979 & 12133 & 12247 \\
\hline \multicolumn{2}{|c|}{$\begin{array}{l}\text { Nombre de personnes entrées: } \\
\text { dans leur première, seconde } \\
\text { année, etc. sur } 23994 \text {. }\end{array}$} & 23994 & 17540 & 15162 & 14177 & 12477 & 12968 & 12562 & 12255 & 12015 & 11861 \\
\hline
\end{tabular}


ANNEXE 2

De la vieillesse et de la mort, in Histoire naturelle..., tome I, 1749, p. 601.

\begin{tabular}{|c|c|c|c|c|c|}
\hline \multicolumn{6}{|c|}{ TABLE DES PROBABILITÉS DE LA DURÉE DE LA VIE } \\
\hline AGE & \multicolumn{2}{|c|}{ DURÉE DE LA VIE } & \multirow{2}{*}{$\begin{array}{c}\text { AGE } \\
\text { ans }\end{array}$} & \multicolumn{2}{|c|}{ DURÉE DE LA VIE } \\
\hline ans & années & mois & & années & mois \\
\hline 0 & 8 & 0 & 43 & 20 & 4 \\
\hline 1 & 33 & 0 & 44 & 19 & 9 \\
\hline 2 & 38 & 0 & 45 & 19 & 3 \\
\hline 3 & 40 & 0 & 46 & 18 & 9 \\
\hline 4 & 41 & 0 & 47 & 18 & 2 \\
\hline 5 & 41 & 6 & 48 & 17 & 8 \\
\hline 6 & 42 & 0 & 49 & 17 & 2 \\
\hline 7 & 42 & 3 & 50 & 16 & 7 \\
\hline 8 & 41 & 6 & 51 & 16 & 0 \\
\hline 9 & 40 & 10 & 52 & 15 & 6 \\
\hline 10 & 40 & 2 & 53 & 15 & 0 \\
\hline 11 & 39 & 6 & 54 & 14 & 6 \\
\hline 12 & 38 & 9 & 55 & 14 & 0 \\
\hline 13 & 38 & 1 & 56 & 13 & 5 \\
\hline 14 & 37 & 5 & 57 & 12 & 10 \\
\hline 15 & 36 & 9 & 58 & 12 & 3 \\
\hline 16 & 36 & 0 & 59 & 11 & 8 \\
\hline 17 & 35 & 4 & 60 & 11 & 1 \\
\hline 18 & 34 & 8 & 61 & 10 & 6 \\
\hline 19 & 34 & 0 & 62 & 10 & 0 \\
\hline 20 & 33 & 5 & 63 & 9 & 6 \\
\hline 21 & 32 & 11 & 64 & 9 & 0 \\
\hline 22 & 32 & 4 & 65 & 8 & 6 \\
\hline 23 & 31 & 10 & 66 & 8 & 0 \\
\hline 24 & 31 & 3 & 67 & 7 & 6 \\
\hline 25 & 30 & 9 & 68 & 7 & 0 \\
\hline 26 & 30 & 2 & 69 & 6 & 7 \\
\hline 27 & 29 & 7 & 70 & 6 & 2 \\
\hline 28 & 29 & 0 & 71 & 5 & 8 \\
\hline 29 & 28 & 6 & 72 & 5 & 4 \\
\hline 30 & 28 & 0 & 73 & 5 & 0 \\
\hline 31 & 27 & 6 & 74 & 4 & 9 \\
\hline 32 & 26 & 11 & 75 & 4 & 6 \\
\hline 33 & 26 & 3 & 76 & 4 & 3 \\
\hline 34 & 25 & 7 & 77 & 4 & 1 \\
\hline 35 & 25 & 0 & 78 & 3 & 11 \\
\hline 36 & 24 & 5 & 79 & 3 & 9 \\
\hline 37 & 23 & 10 & 80 & 3 & 7 \\
\hline 38 & 23 & 3 & 81 & 3 & 5 \\
\hline 39 & 22 & 8 & 82 & 3 & 3 \\
\hline 40 & 22 & 1 & 83 & 3 & 2 \\
\hline 41 & 21 & 6 & 84 & 3 & 1 \\
\hline 42 & 20 & 11 & 85 & 3 & 0 \\
\hline
\end{tabular}


ANNEXE 3

\begin{tabular}{|c|c|c|c|c|c|c|c|c|c|c|}
\hline $\mathrm{A}$ & $\mathrm{B}$ & $\mathrm{C}$ & $\mathrm{D}$ & $\mathrm{E}$ & $F$ & $G$ & $\mathrm{H}$ & I & $\mathrm{J}$ & $\mathrm{K}$ \\
\hline Age & Survivants & $B / 2$ & Mois & Années & Mois & \multicolumn{2}{|c|}{ Buffon } & Écarts & \multicolumn{2}{|c|}{ Nombre de morts } \\
\hline & & & bruts & & arrondis & Années & Mois & & (1749) & (1777) \\
\hline 0 & 23994 & 11997 & 1,403 & 8 & 1 & 8 & 0 & -1 & & \\
\hline 1 & 17540 & 8770 & 0,000 & 33 & 0 & 33 & 0 & 0 & 6454 & 6454 \\
\hline 2 & 15162 & 7581 & 10,337 & 37 & 10 & 38 & 0 & 2 & 2378 & 2378 \\
\hline 3 & 14177 & 7088,5 & 3,672 & 40 & 4 & 40 & 0 & -4 & 985 & 985 \\
\hline 4 & 13477 & 6738,5 & 10,534 & 40 & 11 & 41 & 0 & 1 & 700 & 700 \\
\hline 5 & 12968 & 6484 & 8,250 & 41 & 8 & 41 & 6 & -2 & 509 & 509 \\
\hline 6 & 12562 & 6281 & 1,277 & 42 & 1 & 42 & 0 & -1 & 406 & 406 \\
\hline 7 & 12255 & 6127,5 & 2,195 & 42 & 2 & 42 & 3 & 1 & 307 & 307 \\
\hline 8 & 12015 & 6007,5 & 5,984 & 41 & 6 & 41 & 6 & 0 & 240 & 240 \\
\hline 9 & 11861 & 5930,5 & 8,416 & 40 & 8 & 40 & 10 & 2 & 154 & 154 \\
\hline 10 & 11747 & 5873,5 & 10,216 & 39 & 10 & 40 & 2 & 4 & 114 & 112 \\
\hline 11 & 11666 & 5833 & 11,495 & 38 & 11 & 39 & 6 & 7 & 81 & 100 \\
\hline 12 & 11566 & 5783 & 5,165 & 38 & 5 & 38 & 9 & 4 & 100 & 93 \\
\hline 13 & 11493 & 5746,5 & 10,709 & 37 & 11 & 38 & 1 & 2 & 73 & 88 \\
\hline 14 & 11420 & 5710 & 2,211 & 37 & 2 & 37 & 5 & 3 & 73 & 84 \\
\hline 15 & 11330 & 5665 & 5,763 & 36 & 6 & 36 & 9 & 3 & 90 & 85 \\
\hline 16 & 11233 & 5616,5 & 9,592 & 35 & 10 & 36 & 0 & 2 & 97 & 90 \\
\hline 17 & 11129 & 5564,5 & 2,554 & 35 & 3 & 35 & 4 & 1 & 104 & 95 \\
\hline 18 & 11014 & 5507 & 9,386 & 34 & 9 & 34 & 8 & -1 & 115 & 100 \\
\hline 19 & 10909 & 5454,5 & 3,327 & 34 & 3 & 34 & 0 & -3 & 105 & 107 \\
\hline 20 & 10768 & 5384 & 11,018 & 33 & 11 & 33 & 5 & -6 & 141 & 116 \\
\hline 21 & 10675 & 5337,5 & 1,607 & 33 & 2 & 32 & 11 & -3 & 93 & 124 \\
\hline 22 & 10514 & 5257 & 5,057 & 32 & 5 & 32 & 4 & -1 & 161 & 133 \\
\hline 23 & 10380 & 5190 & 7,929 & 31 & 8 & 31 & 10 & 2 & 134 & 136 \\
\hline 24 & 10259 & 5129,5 & 10,521 & 30 & 11 & 31 & 3 & 4 & 121 & 140 \\
\hline 25 & 10060 & 5030 & 6,000 & 30 & 6 & 30 & 9 & 3 & 199 & 141 \\
\hline 26 & 9926 & 4963 & 0,186 & 30 & 0 & 30 & 2 & 2 & 134 & 142 \\
\hline 27 & 9791 & 4895,5 & 6,465 & 29 & 6 & 29 & 7 & 1 & 135 & 143 \\
\hline 28 & 9640 & 4820 & 1,055 & 29 & 1 & 29 & 0 & -1 & 151 & 144 \\
\hline 29 & 9544 & 4772 & 4,220 & 28 & 4 & 28 & 6 & 2 & 96 & 145 \\
\hline 30 & 9307 & 4653,5 & 0,067 & 28 & 0 & 28 & 0 & 0 & 237 & 148 \\
\hline 31 & 9225 & 4612,5 & 5,533 & 27 & 6 & 27 & 6 & 0 & 82 & 151 \\
\hline 32 & 9045 & 4522,5 & 0,933 & 27 & 1 & 26 & 11 & -2 & 180 & 153 \\
\hline 33 & 8912 & 4456 & 2,427 & 26 & 2 & 26 & 3 & 1 & 133 & 154 \\
\hline 34 & 8780 & 4390 & 3,910 & 25 & 4 & 25 & 7 & 3 & 132 & 158 \\
\hline 35 & 8515 & 4257,5 & 6,888 & 24 & 7 & 25 & 0 & 5 & 265 & 160 \\
\hline 36 & 8328 & 4164 & 8,989 & 23 & 9 & 24 & 5 & 8 & 187 & 165 \\
\hline 37 & 8176 & 4088 & 10,697 & 22 & 11 & 23 & 10 & 11 & 152 & 170 \\
\hline 38 & 8016 & 4008 & 3,259 & 22 & 3 & 23 & 3 & 12 & 160 & 175 \\
\hline 39 & 7929 & 3964,5 & 9,704 & 21 & 10 & 22 & 8 & 10 & 87 & 181 \\
\hline 40 & 7525 & 3762,5 & 0,708 & 22 & 1 & 22 & 1 & 0 & 404 & 187 \\
\hline 41 & 7444 & 3722 & 3,727 & 21 & 4 & 21 & 6 & 2 & 81 & 186 \\
\hline 42 & 7252 & 3626 & 10,882 & 20 & 11 & 20 & 11 & 0 & 192 & 185 \\
\hline 43 & 7124 & 3562 & 3,652 & 20 & 4 & 20 & 4 & 0 & 128 & 184 \\
\hline 44 & 7008 & 3504 & 7,975 & 19 & 8 & 19 & 9 & 1 & 116 & 179 \\
\hline 45 & 6701 & 3350,5 & 5,378 & 19 & 5 & 19 & 3 & -2 & 307 & 172 \\
\hline 46 & 6561 & 3280,5 & 9,162 & 18 & 9 & 18 & 9 & 0 & 140 & 166 \\
\hline 47 & 6449 & 3224,5 & 0,194 & 18 & 0 & 18 & 2 & 2 & 112 & 153 \\
\hline 48 & 6291 & 3145,5 & 4,583 & 17 & 5 & 17 & 8 & 3 & 158 & 159 \\
\hline 49 & 6197 & 3098,5 & 7,194 & 16 & 7 & 17 & 2 & 7 & 94 & 161 \\
\hline
\end{tabular}


ANNEXE 3 (suite)

\begin{tabular}{|c|c|c|c|c|c|c|c|c|c|c|}
\hline $\mathrm{A}$ & $\mathrm{B}$ & $\mathrm{C}$ & $\mathrm{D}$ & $E$ & $\mathrm{~F}$ & $\mathrm{G}$ & $\mathrm{H}$ & I & $\mathrm{J}$ & $\mathrm{K}$ \\
\hline Ace & Survivants & $\mathrm{B} / 2$ & Mois & Annéed & Mois & \multicolumn{2}{|c|}{ Buffon } & Écorts & \multicolumn{2}{|c|}{ Nombre de morts } \\
\hline Age & Survivants & D/2 & bruts & AmInes & arrondis & Années & Mois & Ecarts & $(1749)$ & $(1777)$ \\
\hline 50 & 5817 & 2908,5 & 8,746 & 16 & 9 & 16 & 7 & -2 & 380 & 162 \\
\hline 51 & 5738 & 2869 & 0,052 & 16 & 0 & 16 & 0 & 0 & 79 & 163 \\
\hline 53 & 5586 & 2793 & 4,035 & 15 & 4 & 15 & 6 & 2 & 152 & 164 \\
\hline 53 & 5485 & 2742,5 & 6,681 & 14 & 7 & 15 & 0 & 5 & 101 & 165 \\
\hline 54 & 5375 & 2687,5 & 9,563 & 13 & 10 & 14 & 6 & 8 & 110 & 168 \\
\hline 55 & 5095 & 2547,5 & 11,567 & 14 & 0 & 14 & 0 & 0 & 280 & 170 \\
\hline 56 & 4965 & 2482,5 & 1,937 & 13 & 2 & 13 & 5 & 3 & 130 & 173 \\
\hline 57 & 4836 & 2418 & 3,969 & 12 & 4 & 12 & 10 & 6 & 129 & 174 \\
\hline 58 & 4654 & 2327 & 6,835 & 11 & 7 & 12 & 3 & 8 & 182 & 177 \\
\hline 59 & 4564 & 2282 & 8,252 & 10 & 8 & 11 & 8 & 12 & 90 & 179 \\
\hline 60 & 4030 & 2015 & 1,771 & 11 & 2 & 11 & 1 & -1 & 534 & 183 \\
\hline 61 & 3949 & 1974,5 & 3,565 & 10 & 4 & 10 & 6 & 2 & 81 & 185 \\
\hline 62 & 3772 & 1886 & 7,483 & 9 & 7 & 10 & 0 & 5 & 177 & 186 \\
\hline 63 & 3611 & 1805,5 & 11,048 & 8 & 11 & 9 & 6 & 7 & 161 & 189 \\
\hline 64 & 3450 & 1725 & 6,495 & 8 & 6 & 9 & 0 & 6 & 161 & 190 \\
\hline 65 & 3228 & 1614 & 4,357 & 8 & 4 & 8 & 6 & 2 & 222 & 197 \\
\hline 66 & 3012 & 1506 & 0,047 & 8 & 0 & 8 & 0 & 0 & 216 & 196 \\
\hline 67 & 2870 & 1435 & 3,349 & 7 & 3 & 7 & 6 & 3 & 142 & 195 \\
\hline 68 & 2641 & 1320,5 & 8,674 & 6 & 9 & 7 & 0 & 3 & 229 & 194 \\
\hline 69 & 2544 & 1272 & 10,930 & 5 & 11 & 6 & 7 & 8 & 97 & 191 \\
\hline 70 & 2163 & 1081,5 & 5,350 & 6 & 5 & 6 & 2 & -3 & 381 & 190 \\
\hline 71 & 2055 & 1027,5 & 10,750 & 5 & 11 & 5 & 8 & -3 & 108 & 189 \\
\hline 72 & 1784 & 892 & 10,041 & 5 & 10 & 5 & 4 & -6 & 271 & 188 \\
\hline 73 & 1675 & 837,5 & 6,000 & 5 & 6 & 5 & 0 & -6 & 109 & 187 \\
\hline 74 & 1507 & 753,5 & 2,599 & 5 & 3 & 4 & 9 & -6 & 168 & 181 \\
\hline 75 & 1249 & 624,5 & 8,866 & 4 & 9 & 4 & 6 & -3 & 258 & 177 \\
\hline 76 & 1135 & 567,5 & 11,636 & 4 & 0 & 4 & 3 & 3 & 114 & 175 \\
\hline 77 & 1015 & 507,5 & 11,667 & 4 & 0 & 4 & 1 & 1 & 120 & 174 \\
\hline 78 & 868 & 434 & 10,047 & 3 & 10 & 3 & 11 & 1 & 147 & 170 \\
\hline 79 & 807 & 403,5 & 2,750 & 3 & 3 & 3 & 9 & 6 & 61 & 157 \\
\hline 80 & 560 & 280 & 2,200 & 4 & 2 & 3 & 7 & -7 & 247 & 141 \\
\hline 81 & 506 & 253 & 7,600 & 3 & 8 & 3 & 5 & -3 & 54 & 125 \\
\hline 82 & 420 & 210 & 6,462 & 3 & 6 & 3 & 3 & -3 & 86 & 103 \\
\hline 83 & 348 & 174 & 6,545 & 3 & 7 & 3 & 2 & -5 & 72 & 86 \\
\hline 84 & 291 & 145,5 & 3,767 & 3 & 4 & 3 & 1 & -3 & 57 & 63 \\
\hline 85 & 231 & 115,5 & 0,462 & 3 & 0 & 3 & 0 & 0 & 60 & 54 \\
\hline 86 & 192 & 96 & 2,625 & 3 & 3 & & & & 39 & 44 \\
\hline 87 & 159 & 79,5 & 8,813 & 2 & 9 & & & & 33 & 38 \\
\hline 88 & 116 & 58 & 3,750 & 3 & 4 & & & & 43 & 32 \\
\hline 89 & 103 & 51,5 & 8,625 & 2 & 9 & & & & 13 & 20 \\
\hline 90 & 71 & 35,5 & 7,714 & 3 & 8 & & & & 32 & 18 \\
\hline 91 & 63 & 31,5 & 1,800 & 3 & 2 & & & & 8 & 16 \\
\hline 92 & 47 & 23,5 & 11,400 & 2 & 11 & & & & 16 & 14 \\
\hline 93 & 40 & 20 & 7,200 & 2 & 7 & & & & 7 & 12 \\
\hline 94 & 33 & 16,5 & 9,000 & 2 & 9 & & & & 7 & 10 \\
\hline 95 & 23 & 11,5 & 6,750 & 2 & 7 & & & & 10 & 9 \\
\hline 96 & 18 & 9 & 10,500 & 1 & 11 & & & & 5 & 7 \\
\hline 97 & 16 & 8 & 0,000 & 1 & 0 & & & & 2 & 5 \\
\hline 98 & 8 & 4 & 5,143 & 1 & 5 & & & & 8 & 4 \\
\hline 99 & 7 & 3,5 & 6,000 & 0 & 6 & & & & 1 & 3 \\
\hline
\end{tabular}


ANNEXE 3 (suite)

\begin{tabular}{|c|c|c|c|c|c|c|c|c|c|c|c|}
\hline A & B & $\mathrm{C}$ & $\mathrm{D}$ & $\mathrm{E}$ & $\mathrm{F}$ & $\mathrm{G}$ & $\mathrm{H}$ & $\mathrm{I}$ & $\mathrm{J}$ & $\mathrm{K}$ \\
\hline Age & Survivants & $\mathrm{B} / 2$ & $\begin{array}{c}\text { Mois } \\
\text { bruts }\end{array}$ & Années & Mois & \multicolumn{2}{c|}{ Buffon } & ácarts & \multicolumn{2}{c|}{$\begin{array}{c}\text { Nombre de morts } \\
(1749)\end{array}$} & $(1777)$ \\
\hline 100 & & & & & & & & & 7 & 5 \\
\hline 101 & & & & & & & & & & 2 \\
\hline 102 & & & & & & & & & & 0 \\
\hline
\end{tabular}

La colonne B indique le nombre de survivants tel qu'il est donné par Buffon pour chaque année ; la colonne $\mathrm{C}$ la moitié de celui-ci (les nombres figurant dans la colonne $\mathrm{B}$ corrigent les coquilles que comportait l'édition de 1749, puisque Buffon a visiblement calculé les vies probables sur les chiffres exacts. Ainsi la $4^{\mathrm{e}}$ année donnait dans l'édition originale, comme le signalera Buffon lui-même en 1877, 12477 et non 13477, ce qui est visiblement une erreur, et aurait porté la vie probable à cet âge à 44 ans et 7 mois).

Le calcul décrit ci-dessous permet de déterminer le nombre de mois qu'il convient d'ajouter à chaque année, d'abord tel qu'il résulte directement du calcul (colonne D), puis arrondi (colonne F). Les colonnes $\mathrm{E}$ et $\mathrm{F}$ offrent donc les vies probables obtenues par la méthode décrite ci-dessous, les colonnes $\mathrm{G}$ et $\mathrm{H}$ rappellent celles que donne Buffon dans l'article de 1749.

Mode de calcul de la vie probable (colonnes E et F) : il s'agit de trouver la vie médiane $V_{n}$ pour un âge $A_{n}$ (colonne A) auquel correspond un demi-effectif de survivants $C_{n}$ (colonne C). Le calcul s'effectue en 3 temps :

$\left.1^{\circ}\right)$ Calcul du nombre d'années : on détermine l'âge en année en recherchant $A_{x}$, correspondant à $B_{x}$ tel que $B_{x}>C_{n}>B_{x+1}$.

$2^{\circ}$ ) Calcul du nombre de mois : la différence $B_{x}-B_{x+1}$ correspondant par définition à 12 mois, on calcule $B_{x}-B_{n}$ qui correspond à $\mathrm{N}$ mois. On calcule alors $\mathrm{N}=\frac{12 \square\left(B_{x} \square C_{n}\right)}{B_{x} \square B_{x+1}}$ (colonne D).

$\left.3^{\circ}\right)$ En réduisant $\mathrm{N}$ à sa partie entière (colonne $\left.\mathrm{F}\right)$, on en déduit $V_{n}=\left(A_{x}-A_{n}\right)$ années (colonne $\mathrm{E}$ ) et $\mathrm{N}$ mois (colonne $\mathrm{F}$ ).

Les colonnes $\mathrm{J}$ et $\mathrm{K}$ du tableau fournissent le décompte du nombre de morts par année tel qu'il est calculé par Buffon respectivement en 1749 (colonne J) et en 1777 (colonne K). 


\section{ANNEXE 4 \\ Des probabilités de la durée de la vie, in Histoire naturelle..., Supplément, tome IV, 1777, pp. 151-152.}

«Il n’y a point de correction à faire jusqu'au nombre 154, qui correspond à la neuvième année, parce qu'on ne se trompe guère d'un an sur l'âge d'un enfant de $1,2,3$, $4,5,6,7$ ou 8 ans : mais le nombre 114, qui correspond à la dixième année, est trop fort, aussi bien que le nombre 100, qui correspond à la douzième ; tandis que le nombre 81 , qui correspond à la onzième, est trop faible. Le seul moyen de rectifier ces défauts et ces excès, et d'approcher de la vérité, c'est de prendre les nombres cinq à cinq, et de les partager de manière qu'ils augmentent proportionnellement à mesure que leurs sommes vont en augmentant; et au contraire, de les partager de manière qu'ils aillent en diminuant si leurs sommes vont aussi en diminuant : par exemple, j'ajoute ensemble les cinq nombres $114,81,100,73$ et 73 , qui correspondent dans la table à la $10^{\mathrm{e}}, 11^{\mathrm{e}}, 12^{\mathrm{e}}, 13^{\mathrm{e}}$ et $14^{\mathrm{e}}$ année, leur somme est 441 ; je partage cette somme d'abord en cinq parties égales, ce qui me donne $88 \frac{1}{5}$. J'ajoute de même les cinq nombres suivants 90, 97, 104, 115 et 105, leur somme est 511, et je vois par-là que ces sommes vont en augmentant; dès lors je partage la somme 441 des cinq nombres précédents, en sorte qu'ils aillent en augmentant, et j'écris 87, 87, 88, 89 et 90, au lieu de 114,81, 100, 73 et 73. De même, avant de partager la somme 511 des cinq nombres 90,97, 104, 115 et 105, qui correspondent à la $15^{\mathrm{e}}, 16^{\mathrm{e}}$, $17^{\mathrm{e}} 18^{\mathrm{e}}$ et $19^{\mathrm{e}}$ année, j'ajoute ensemble les cinq nombres suivants, pour voir si leur somme est plus ou moins forte que 511 : et comme je la trouve plus forte, je partage 511 , comme j'ai partagé 441 en cinq parties qui aillent en augmentant; et si au contraire cette somme des cinq nombres suivants était plus petite que celle des cinq nombres précédents (comme cela se trouve dans la suite), je partagerais cette somme de manière que les nombres aillent en diminuant. De cette façon, nous approcherons de la vérité autant qu'il est possible, d'autant que je ne me suis déterminé à commencer mes corrections au terme 114 , qu' après avoir tâtonné toutes les autres suites que donnaient les sommes des nombres pris cinq à cinq et même dix à dix, et que c'est à ce terme que je me suis fixé, parce que leur marche s'est trouvée avoir le plus d'uniformité».

\section{ANNEXE 5}

A. Deparcieux, Addition à l'Essai sur les probabilités de la durée de la vie humaine. Paris, Guerin \& Delatour, 1760, pp. 22-23.

Ayant rappelé la remarque de Buffon à propos des nombres ronds, Deparcieux ajoute : «On sent bien que la Nature ne fait pas de pareils sauts, et qu' on ne peut pas parier, par exemple, que de 8712 personnes qui naissent, il en mourra 46 à l'âge de 49 ans, 159 à l'âge de 50 ans et 63 à l'âge de 51 ans ; mais on pourrait assez probablement parier qu'il en mourra 344 dans les cinq années de 48 à 52 ans; au moins serait-on beaucoup plus près de la vérité ; et si on divise le nombre, 344 , qui est la somme de ce que les Registres ont donné pour ces cinq années, de 48 à 52, en cinq parties, telles que la première et la dernière quadrent, par les différences, avec la plus basse de celles d'audessus, et la plus haute de celles d'au-dessous (supposées distribuées de même), on formera un ordre de mortalité, tel que la Nature ne s'en écartera pas de beaucoup; et que si elle s'en écarte, ce sera aussi souvent en plus qu'en moins, ou en moins qu'en plus». 


\section{ANNEXE 6}

Histoire naturelle..., Supplément, tome IV, 1777, p. 158.

TABLE DE LA PROBABILITÉ DE LA VIE

Pour un enfant qui vient de naître.

On peut parier :

- 17540 contre 6454 , ou, pour abréger, $2 \frac{3}{4}$ environ contre 1 , qu'un enfant qui vient de naître vivra un an. En en supposant la mort également répartie dans tout le courant de l'année :

- 17540 contre $\frac{6454}{2}$, ou $5 \frac{7}{16}$ contre 1 , qu'il vivra 6 mois ;

- 17540 contre $\frac{6454}{2}$, ou près de 11 contre 1 , qu'il vivra 3 mois ; et

- 17540 contre $\frac{6454}{565}$, ou environ 1030 contre 1 qu'il ne mourra pas dans les vingt-quatre heures.

De même on peut parier :

- 14162 contre 8832 , ou 1 environ contre $1 \frac{5}{4}$, qu'un enfant qui vient de naître vivra 2 ans ;

- 14117 contre 9817 , ou $1 \frac{4}{9}$ contre 1 , qu'il vivra 3 ans ;

- 13477 contre 10517 , ou $1 \frac{1}{5}$ contre 1 , qu'il vivra 4 ans ;

- 12968 contre 11026 , ou $1 \frac{2}{11}$ contre 1 , qu'il vivra 5 ans ;

- 12562 contre 11432 , ou $1 \frac{1}{11}$ contre 1 , qu'il vivra 6 ans ;

- 12255 contre 11739 , ou $1 \frac{1}{25}$ environ contre 1 , qu'il vivra 7 ans ;

- 12015 contre 11979 , ou $1 \frac{1}{555}$ contre 1 , qu'il vivra 8 ans ;

- 12133 contre 11861 , ou $1 \frac{1}{45}$ contre 1 , qu'il ne vivra pas 9 ans ;

- 12245 contre 11749 , ou $1 \frac{1}{24}$ contre 1 , qu'il ne vivra pas 10 ans ;

- 12345 contre 11649 , ou $1 \frac{1}{17}$ contre 1 , qu'il ne vivra pas 11 ans ;

- 12438 contre 11556 , ou $1 \frac{1}{15}$ contre 1 , qu'il ne vivra pas 12 ans ;

- 12526 contre 11468 , ou $1 \frac{1}{11}$ contre 1 , qu'il ne vivra pas 13 ans ;

- 12610 contre 11384 , ou $1 \frac{1}{9}$ contre 1 , qu'il ne vivra pas 14 ans ;

- 12695 contre 11299 , ou $1 \frac{1}{8}$ contre 1 , qu'il ne vivra pas 15 ans ;

- 12785 contre 11209 , ou $1 \frac{1}{7}$ contre 1 , qu'il ne vivra pas 16 ans ;

- 12880 contre 11114 , ou $1 \frac{1}{6}$ contre 1 , qu'il ne vivra pas 17 ans ;

- 12980 contre 11014 , ou $1 \frac{2}{11}$ contre 1 , qu'il ne vivra pas 18 ans ;

- 13087 contre 10907 , ou $1 \frac{1}{5}$ contre 1 , qu'il ne vivra pas 19 ans ; 


\section{ANNEXE 7 \\ Histoire naturelle..., Supplément, tome IV, 1777.}

Plan du texte des pages 265-323.

Remarque : Nous indiquons entre crochets l'effectif de naissances et de morts pris en compte par chacune de ces études.

I. «État général des naissances, des mariages et des morts dans la ville de Paris, depuis l'année 1709 jusques et compris l'année 1766 inclusivement», pp. 265-266, [N : 1074367, M : 1087959].

II. État des baptêmes, mariages et morts pour Paris et faubourgs, comptés par mois pour chacune des années de la période 1745-1766, pp. 267-285, [N : 416201, M : 413551].

III. «État des baptêmes, mariages et sépultures dans la ville de Montbard en Bourgogne, depuis 1765 inclusivement, jusques et compris l'année 1774», pp. 286-288, [N : 826, M : $651]$.

IV. «État des baptêmes, mariages et morts dans la ville de Semur en Auxois, depuis 1770 jusques et compris l'année 1774», pp. 289-290, [N : 776, M : 622].

V. «État des baptêmes, mariages et morts dans la petite ville de Flavigny, depuis 1770 jusques et compris l'année 1774», pp. 290-291, [N : 185, M : 162].

VI. «État des baptêmes, mariages et morts dans la petite ville de Vitteaux, depuis 1770 jusques et compris l'année 1774», pp. 291-292, [N : 415, M : 308].

VII. «État des baptêmes, mariages et morts dans le bourg d'Époisses, et dans les villages de Genay, Marigny-le-Grand et Toutry, bailliage de Semur-en-Auxois, depuis 1770 jusques et compris l'année 1774, avec leur population actuelle», pp. 293-294, [N : 491, $\mathrm{M}: 578]$.

VIII. «État des baptêmes, mariages et morts dans le bailliage entier de Semur-en-Auxois, contenant 99, tant villes que bourgs et villages, pour les années depuis 1770 jusques et compris l'année 1774», pp. 294-298, [N : 8165, M : 6408].

IX. «État des baptêmes, mariages et morts dans le bailliage de Saulieu-en-Bourgogne, contenant 40, tant villes que bourgs et villages, pour les années depuis 1770 jusques et compris l'année 1772», pp. 299-303, [N : 3043, M : 2218 ; l'étude porte en fait sur 39 paroisses].

$\mathrm{X}$. «Comparaison de la mortalité à Paris, et dans les campagnes à dix, quinze et vingt lieues de distance de cette ville», pp. 303-306, [pour un effectif de 13189 individus, soit le nombre des morts pour les trois paroisses parisiennes de la table de Dupré de SaintMaur].

XI. «Comparaison de tables de mortalité en France avec les tables de la mortalité à Londres», pp. 306-323, [d'après les tables de Corbyn-Morris pour la période 1728-1757]. 


\section{BIBLIOGRAPHIE}

D'ALEMBERT, J., «Note sur le mémoire Sur l'application du calcul des probabilités à l'inoculation de la petite vérole» (1 $1{ }^{\mathrm{e}}$ mémoire), in Opuscules mathématiques, Paris, David, tome II, 1761, pp. 47-95.

D'ALEMBERT, J., «Sur l'analyse des jeux», Opuscules mathématiques, Paris, David, tome IV, 1768, pp. 80-92.

D'ALEMBERT, J., «Sur la durée de la vie», in Opuscules mathématiques, Paris, David, tome IV, 1768, pp. 92-98.

BADET, L., «Buffon, précurseur de la science démographique», Annales de géographie, tome XXXVII, 1929, pp. 206-220.

BEHAR, L., «Des tables de mortalité aux XVII ${ }^{\mathrm{e}}$ et $\mathrm{XVIII}^{\mathrm{e}}$ siècles. Histoire Signification», Annales de démographie historique, 1976, pp. 173-200.

BUFFON, G., «Préface du traducteur», in NEWTON, La Méthode des Fluxions et des suites infinies, Paris, Debure l'aîné, 1740, pp. iii-xxx.

BUFFON, G., «Formule sur les échelles arithmétiques», in Mémoires de l'Académie Royale des Sciences de Paris, année 1741, Paris, 1744, pp. 219-222.

BUFFON, G., «Sur la conservation et le rétablissement des forêts», MARSP, année 1739, Paris, 1741, pp. 140-156 ; Histoire naturelle, générale et particulière, servant de suite à la Théorie de la terre, Supplément, tome second, Paris, Imprimerie Royale, 1775, pp. 249271.

BUFFON, G., «Sur la culture et l'exploitation des forêts», MARSP, année 1742, Paris, 1745, pp. 233-246 ; Histoire naturelle, générale et particulière, servant de suite à la Théorie de la terre, Supplément, tome second, Paris, Imprimerie Royale, 1775, pp. 271290.

BUFFON, G., «De la vieillesse et de la mort», Histoire naturelle de l'Homme, in Histoire naturelle, générale et particulière, Paris, Imprimerie royale, tome second, 1749, pp. 557603.

BUFFON, G., «De la manière d'étudier et de traiter l'histoire naturelle», in Histoire naturelle, générale et particulière, Paris, Imprimerie royale, tome premier, 1749 ; in Euvres philosophiques de Buffon, édité par Jean Piveteau, Paris, Presses Universitaires de France, coll. du Corpus général des philosophes français, 1954, pp. 7-26.

BUFFON, G., Essai d'arithmétique morale, in Histoire naturelle, générale et particulière, servant de suite à l'Histoire naturelle de l'Homme, Supplément, tome quatrième, Paris, Imprimerie Royale, 1777, pp. 46-148.

BUFFON, G., Des probabilités de la durée de la vie, in Histoire naturelle, générale et particulière, servant de suite à l'Histoire naturelle de l'Homme, Supplément, tome quatrième, Paris, Imprimerie Royale, 1777, pp. 149-264. 
BUFFON, G., «État général des naissances, des mariages et des morts...» et articles suivants, in Histoire naturelle, générale et particulière, servant de suite à l'Histoire naturelle de l'Homme, Supplément, tome quatrième, Paris, Imprimerie Royale, 1777, pp. 265-323.

CANGUILHEM, G., La connaissance de la vie, Paris, Hachette, 1952, 2éd. Vrin, 1975.

CONDORCET, N., «Arithmétique politique», Encyclopédie méthodique, Mathématiques, Paris, Panckoucke, t. I, 1784, pp. 135a-136a.

CONDORCET, N., «Éloge de M. le Comte de Buffon», in Histoire de l'Académie Royale des Sciences, 1791 pour 1788 ; in CEuvres de Condorcet, F. Arago et A. O'Connor (éd.), Paris, Firmin-Didot, 1847-1849, 12 vol., tome III, pp. 345-348.

CONDORCET, N., Arithmétique politique. Textes rares ou inédits (1767-1789), édité par B. Bru et P. Crépel, Paris, I.N.E.D., 1994.

COURNOT, A.A., Considérations sur la marche des idées et des événements dans les temps modernes, Paris, 1872, rééd. Paris, Vrin, 1973.

DIDEROT, D., «Arithmétique politique», Encyclopédie méthodique, Mathématiques, Paris, Panckoucke, t. I, 1784, pp. 132b-135a.

DUPAQUIER, J. et M., Histoire de la démographie. La statistique de la population des origines à 1914, Paris, Librairie Académique Perrin, 1985.

DUPAQUIER, J., «Londres ou Paris ? Un grand débat dans le petit monde des arithméticiens politiques (1662-1759)», Population, janvier-avril 1998, n 1-2, pp. 311325.

FONTAINE DES BERTINS, Mémoires donnés à l'Académie Royale des Sciences, non imprimés dans leur temps, Paris Imprimerie Royale, 1764.

FRECHET, M., «Buffon, philosophe des mathématiques», Bulletin de l'institut d'Égypte, $\mathrm{n}^{\circ}$ 28, 1954, pp. 185-202, puis «Buffon comme philosophe des mathématiques», in Cuvres philosophiques de Buffon, édité par Jean Piveteau, Paris, Presses Universitaires de France, 1954, pp. 435-446; repris sous le titre «Buffon comme mathématicien, statisticien et philosophe des mathématiques» dans Les mathématiques et le concret, Paris, Presses Universitaires de France, 1955, pp. 344-367.

HANKS, L., Buffon avant l'«Histoire Naturelle», Paris, Presses Universitaires de France, 1966.

MARTIN, T., «Certitude et probabilité selon Buffon», L'Enseignement philosophique, $49^{\mathrm{e}}$ année, $\mathrm{n}^{\circ} 3$, janvier-février 1999, pp. 5-18.

YOUNG, A., Arithmétique politique, trad. M. Freville, La Haye, 1775, 2 vol. 